\title{
FACTOR SPACES AND IMPLICATIONS \\ ON KIRCHHOFF EQUATIONS WITH \\ CLAMPED BOUNDARY CONDITIONS
}

\author{
IRENA LASIECKA AND ROBERTO TRIGGIANI
}

Received 18 June 2001

We consider mixed problems for the Kirchhoff elastic and thermoelastic systems, subject to boundary control in the clamped boundary conditions BC (clamped control). If $w$ denotes the elastic displacement and $\theta$ the temperature, we establish sharp regularity of $\left\{w, w_{t}, w_{t t}\right\}$ in the elastic case, and of $\left\{w, w_{t}, w_{t t}, \theta\right\}$ in the thermoelastic case. Our results complement those by Lagnese and Lions (1988), where sharp (optimal) trace regularity results are obtained for the corresponding boundary homogeneous cases. The passage from the boundary homogeneous cases to the corresponding mixed problems involves a duality argument. However, in the present case of clamped BC, and only in this case, the duality argument in question is both delicate and technical. In this respect, the clamped BC are "exceptional" within the set of canonical BC (hinged, clamped, free BC). Indeed, it produces new phenomena which are accounted for by introducing new, untraditional factor (quotient) spaces. These are critical in describing both interior regularity and exact controllability of mixed elastic and thermoelastic Kirchhoff problems with clamped controls.

\section{Introduction, motivation, statement of main results on regularity of Kirchhoff systems with clamped boundary controls}

The main goal of this paper is to provide sharp, in fact optimal, regularity results of mixed problems involving Kirchhoff elastic and thermoelastic systems, with control acting in the clamped boundary conditions (BC). The corresponding sharp trace regularity results for the corresponding homogeneous Kirchhoff elastic and thermoelastic systems are already available in the literature [7, pages $123,157-158]$. However, the passage — by duality or transposition-from the latter homogeneous problem in [7] to the former mixed problem given here 
is both delicate and technical. In this respect, the clamped BC are "exceptional" within the set of canonical BC (hinged, clamped, free BC). As we will see, this passage will require first, the introduction of untraditional, new function spaces (called $\tilde{L}_{2}(\Omega)$ and $\tilde{H}_{-1}(\Omega)$ see (2.29) and (3.35) below); next, the study of their properties (in particular, their key characterizations as appropriate factor, or quotient, spaces, given in Propositions 2.7 and 3.3, respectively, along with the identity in (3.38)); finally, some untraditional and nonstandard dualities, dictated by the intrinsic underlying spaces. Key regularity results of the present paper follow.

\subsection{The elastic and thermoelastic mixed problems}

Elastic Kirchhoff equation. Let $\Omega$ be an open bounded domain in $\mathbb{R}^{n}$ with smooth boundary $\Gamma$. Consider the following Kirchhoff elastic mixed problem with clamped boundary control in the unknown $w(t, x)$ :

$$
\begin{array}{ll}
w_{t t}-\gamma \Delta w_{t t}+\Delta^{2} w=0 & \text { in }(0, T] \times \Omega \equiv Q ; \\
w(0, \cdot)=w_{0}, \quad w_{t}(0, \cdot)=w_{1} & \text { in } \Omega \\
\left.w\right|_{\Sigma} \equiv 0,\left.\quad \frac{\partial w}{\partial v}\right|_{\Sigma} \equiv u & \text { in }(0, T] \times \Gamma \equiv \Sigma .
\end{array}
$$

In (1.1a), $\gamma$ is a positive constant to be kept fixed throughout this paper, $\gamma>0$. When $n=2$, problem (1.1) describes the evolution of the displacement $w$ of the elastic Kirchhoff plate model, which accounts for rotational inertia. In it, the constant $\gamma$ is proportional to the square of the thickness of the plate $[7,8]$.

Thermoelastic Kirchhoff equations. With $\Omega, \Gamma$, and $\gamma>0$ as above, consider now the corresponding thermoelastic mixed problem with clamped boundary control in the unknown $\{w(t, x), \theta(t, x)\}$

$$
\begin{array}{ll}
w_{t t}-\gamma \Delta w_{t t}+\Delta^{2} w+\Delta \theta=0 & \text { in }(0, T] \times \Omega \equiv Q \\
\theta_{t}-\Delta \theta-\Delta w_{t}=0 & \text { in } Q ; \\
w(0, \cdot)=w_{0}, \quad w_{t}(0, \cdot)=w_{1}, \quad \theta(0, \cdot)=\theta_{0} & \text { in } \Omega ; \\
\left.w\right|_{\Sigma} \equiv 0 ;\left.\quad \frac{\partial w}{\partial v}\right|_{\Sigma} \equiv u ;\left.\quad \theta\right|_{\Sigma} \equiv 0 & \text { in }(0, T] \times \Gamma \equiv \Sigma
\end{array}
$$

Again, when $n=2$, problem (1.2) describes the evolution of the displacement $w$ and of the temperature $\theta$ (with respect to the stress-free temperature) of the thermoelastic Kirchhoff plate model, which accounts for rotational inertia [7, 8].

1.2. Statement of main results: optimal interior regularity. The following results provide optimal regularity properties for the mixed problems (1.1) and 
(1.2). They justify the introduction of the spaces $\tilde{L}_{2}(\Omega)$ and $\tilde{H}_{-1}(\Omega)$ in Sections $2.2,2.3,2.4$, and 3 , respectively.

THEOREM 1.1. Consider the Kirchhoff elastic problem (1.1) with $\left\{w_{0}, w_{1}\right\}=0$ subject to the hypothesis that

$$
u \in L_{2}\left(0, T ; L_{2}(\Gamma)\right) \equiv L_{2}(\Sigma)
$$

Then, continuously,

$$
\begin{gathered}
w \in C\left([0, T] ; H_{0}^{1}(\Omega)\right) ; \\
w_{t} \in C\left([0, T] ; \tilde{L}_{2}(\Omega)\right) ; \\
w_{t t} \in L_{2}\left(0, T ; \tilde{H}_{-1}(\Omega)\right) .
\end{gathered}
$$

Theorem 1.1 is proved in Section 4.5. A complementary surjectivity result is given in Theorem 1.4, after the introduction of the preliminary material.

THEOREM 1.2. Consider the Kirchhoff thermoelastic problem (1.2) with $\left\{w_{0}, w_{1}\right.$, $\left.\theta_{0}\right\}=0$, subject to the same hypothesis (1.3) on $u$. Then, the map

$$
\begin{aligned}
& u \in L_{2}\left(0, T ; L_{2}(\Gamma)\right) \Longrightarrow\left\{w, w_{t}\right\} \in C\left([0, T] ; H_{0}^{1}(\Omega) \times \tilde{L}_{2}(\Omega)\right) \\
& \Longrightarrow\left[w_{t t}-\frac{1}{\gamma} \theta\right] \in L_{2}\left(0, T ; \tilde{H}_{-1}(\Omega)\right) \\
& \Longrightarrow \theta \in L_{p}\left(0, T ; H^{-1}(\Omega)\right) \cap C\left([0, T] ; H^{-1-\epsilon}(\Omega)\right), \\
& 1<p<\infty ; \forall \epsilon>0,
\end{aligned}
$$

is continuous. However, in addition

$$
\begin{aligned}
& \theta \in C\left([0, T] ; L_{2}(\Omega)\right) \text {, and } w_{t t} \in L_{2}\left(0, T ; \tilde{H}_{-1}(\Omega)\right), \text { but not } \\
& \text { continuously in } u \in L_{2}\left(0, T ; L_{2}(\Gamma)\right) .
\end{aligned}
$$

Theorem 1.2 is proved in Section 5.1. The regularity of $\left\{w, w_{t}\right\}$ in Theorem 1.2 is sharp. As to the regularity of $\theta$, an alternative complementary result, which neither contains Theorem 1.2, (1.8) and (1.9), nor is contained by it, is as follows.

THEOREM 1.3. With reference to the Kirchhoff thermoelastic problem (1.2) with $\left\{w_{0}, w_{1}, \theta_{0}\right\}=0$, then

$$
\theta(t)=-w_{t}(t)+\theta_{1}(t)
$$


where $w_{t}$ satisfies (1.6) of Theorem 1.2, while $\theta_{1}$ satisfies the following property: the map

$$
\begin{aligned}
& u \in L_{2}\left(0, T ; L_{2}(\Gamma)\right) \\
& \longrightarrow \theta_{1}(t)=\int_{0}^{t} e^{-\mathscr{A}(t-\tau)} w_{t t}(\tau) d \tau \in C\left([0, T] ;\left[H_{00}^{1 / 2}(\Omega)\right]^{\prime}\right)
\end{aligned}
$$

is continuous, where $H_{00}^{1 / 2}(\Omega)=\mathscr{D}\left(\mathscr{A}^{1 / 4}\right)$ (see [19, page 66]).

A sketch of the proof of Theorem 1.3 is given in Section 5.2.

\subsection{Literature}

Kirchhoff elastic problem (1.1). With reference, at first, to the homogeneous Kirchhoff system

$$
\begin{array}{ll}
\phi_{t t}-\gamma \Delta \phi_{t t}+\Delta^{2} \phi=0 & \text { in }(0, T] \times \Omega \equiv Q ; \\
\phi(T, \cdot)=\phi_{0}, \quad \phi_{t}(T, \cdot)=\phi_{1} & \text { in } \Omega ; \\
\left.\phi\right|_{\Sigma} \equiv 0 ;\left.\quad \frac{\partial \phi}{\partial v}\right|_{\Sigma} \equiv 0 & \text { in }(0, T] \times \Gamma \equiv \Sigma
\end{array}
$$

where

$$
\left\{\phi_{0}, \phi_{1}\right\} \in H_{0}^{2}(\Omega) \times H_{0}^{1}(\Omega),
$$

sharp trace estimates were obtained in [7]. More precisely, [7] establishes, by multiplier techniques, both of the following results:

(i) the trace regularity inequality for any $T>0$,

$$
\int_{0}^{T} \int_{\Gamma}|\Delta \phi|^{2} d \Sigma \leq c_{T}\left\|\left\{\phi_{0}, \phi_{1}\right\}\right\|_{H_{0}^{2}(\Omega) \times H_{0}^{1}(\Omega)}^{2},
$$

(see [7, equation (2.2.4), page 123]), for some constant $c_{T}>0$,

(ii) the continuous observability inequality, for all $T>$ some $T_{0}>0$,

$$
\begin{aligned}
c(T- & \left.T_{0}\right)\left\|\left\{\phi_{0}, \phi_{1}\right\}\right\|_{H_{0}^{2}(\Omega) \times H_{0}^{1}(\Omega)}^{2} \\
& \leq \int_{0}^{T} \int_{\Gamma\left(x_{0}\right)}|\Delta \phi|^{2} d \Sigma, \quad c>0,
\end{aligned}
$$

(see [7, equation (2.2.3), page 123]). Here, $T_{0}$ is a suitable positive constant, depending on $\gamma>0$, as well as the domain, and $\Gamma\left(x_{0}\right)=\{x \in$ $\left.\Gamma:\left(x-x_{0}\right) \cdot v(x) \geq 0\right\}$, where $v(x)=$ unit outward normal at $x \in \Gamma$.

As is well known, it is a common duality or transposition argument that converts, as usual, inequalities such as (1.14) and (1.15), into, respectively: 
(a) an interior regularity result $u \rightarrow\left\{w, w_{t}\right\}$ of the $w$-problem (1.1) (see [12]);

(b) an exact controllability result (surjectivity or ontoness of the map)

$$
u \in L_{2}\left(0, T ; L_{2}\left(\Gamma\left(x_{0}\right)\right)\right) \longrightarrow\left\{w(T), w_{t}(T)\right\}
$$

onto a suitable state space (see $[11,12])$.

However, in the present case, the duality or transposition argument is nonstandard, due to the special function spaces involved related to the $\mathrm{BC}$. The details, taken from $[4,9,22]$, are given in Section 4.5 in a systematic functional analytic treatment. Here, we carry out a PDE-version of the transposition argument to deduce the interior regularity $u \rightarrow\left\{w, w_{t}\right\}$ in (a).

$P D E$-version of duality argument. Multiplying the nonhomogeneous $w$-problem (1.1) with $\left\{w_{0}, w_{1}\right\}=0$ and $u \in L_{2}\left(0, T ; L_{2}(\Gamma)\right)$ by the solution $\phi$ of problem (1.12), we obtain after integration by parts in $t$, and we use of Green's second theorem, once the appropriate boundary conditions (1.1c) and (1.12c) are invoked:

$$
\begin{aligned}
0= & \int_{0}^{T}\left((1-\gamma \Delta) w_{t t}, \phi\right)_{\Omega} d t+\int_{0}^{T}\left(\Delta^{2} w, \phi\right)_{\Omega} d t \\
= & {\left[\left((1-\gamma \Delta) w_{t}, \phi\right)_{\Omega}\right]_{0}^{T}-\left[\left((1-\gamma \Delta) w, \phi_{t}\right)_{\Omega}\right]_{0}^{T} } \\
& +\int_{0}^{T}\left((1-\gamma \Delta) w, \phi_{t t}\right)_{\Omega} d t+\int_{0}^{T}\left(w, \Delta^{2} \phi\right)_{\Omega} d t \\
& +\int_{0}^{T}\left(\frac{\partial w}{\partial v}, \Delta \phi\right)_{\Gamma} d t,
\end{aligned}
$$

where ( , ) denotes $L_{2}(\Omega)$ or $L_{2}(\Gamma)$-norms. In the first integral term on the right of (1.17), $(1-\gamma \Delta)$ may be moved from the left (as acting on $w$ ) to the right (as acting on $\phi_{t t}$ ) by Green's theorem, with no boundary terms by (1.12c), after which the use of (1.12a) makes the sum of the first two integral terms on the right of (1.17) vanish. Finally, this and (1.1c) yield from (1.17)

$$
\begin{aligned}
& \left((1-\gamma \Delta) w_{t}(T), \phi(T)\right)_{L_{2}(\Omega)} \\
& \quad-\left((1-\gamma \Delta) w(T), \phi_{t}(T)\right)_{L_{2}(\Omega)}+\int_{0}^{T}\left(u,\left.\Delta \phi\right|_{\Gamma}\right)_{\Gamma} d t=0 .
\end{aligned}
$$

The boundary integral term in (1.18) is well defined by $u$ in (1.3) and $\left.\Delta \phi\right|_{\Gamma}$ in (1.14). Thus, we need to investigate the well-posedness of the terms involving the initial conditions

$$
\left((1-\gamma \Delta) w_{t}(T), \phi(T)\right)_{L_{2}(\Omega)}, \quad\left((1-\gamma \Delta) w(T), \phi_{t}(T)\right)_{L_{2}(\Omega)}
$$


As $\phi_{t}(T) \in H_{0}^{1}(\Omega)$ by (1.13), the well-posedness of the second term in (1.19) then requires

$$
(1-\gamma \Delta) w(T)=\mathscr{A}_{\gamma} w(T) \in H^{-1}(\Omega) \equiv\left[\mathscr{D}\left(\mathscr{A}_{\gamma}^{1 / 2}\right)\right]^{\prime},
$$

invoking the operator $\mathscr{A}_{\gamma}$ in (2.2) below (since $w(T)$ satisfies zero Dirichlet $\mathrm{BC}$, as in (1.1c)); or finally

$$
w(T) \in \mathscr{D}\left(\mathscr{A}_{\gamma}^{1 / 2}\right) \equiv H_{0}^{1}(\Omega)
$$

So far, all is essentially standard. Not so for the first term of (1.19), however. Indeed, as $\phi(T) \in H_{0}^{2}(\Omega)$ by (1.13), the well-posedness of the first term in (1.19) then requires

$$
(1-\gamma \Delta) w_{t}(T)=\mathscr{A}_{\gamma} w_{t}(T) \in H^{-2}(\Omega) \equiv\left[\mathscr{D}\left(A^{1 / 2}\right)\right]^{\prime},
$$

invoking the operator $\mathscr{A}_{\gamma}$ in (2.2) below (since $w_{t}(T)$ satisfies zero Dirichlet BC, by (1.1c)), as well as the elastic operator $A$ in (2.1) and (2.3) below. Thus, (1.22) characterizes $w_{t}(T)$ as satisfying the condition

$$
A^{-1 / 2} A_{\gamma} w_{t}(T) \in L_{2}(\Omega)
$$

But (1.23), in turn, characterizes $w_{t}(T)$ as belonging to the space which we call $\tilde{L}_{2}(\Omega)$ in Section 2.2, (2.29), see its characterization (2.33). We conclude that

$$
w_{t}(T) \in \tilde{L}_{2}(\Omega)
$$

This conclusion was already noted in [9], via, however, a functional analytic (rather than PDE's) approach, such as the one in Section 4.5 below, but the space $\tilde{L}_{2}(\Omega)$ was not clarified there beyond its definition (2.29) below. In particular, the characterization (2.49) of Proposition 2.7 is a new result of the present paper. [9] was motivated by [7], where the space $\tilde{L}_{2}(\Omega)$ for $w_{t}$ does not appear. The point that we wish to make is that it is the space $\tilde{L}_{2}(\Omega)$ (not $L_{2}(\Omega)$ ) that describes the optimal regularity — as well as the controllability — of the velocity $w_{t}$ of the mixed problem (1.1).

Surjectivity. Thus conclusions (1.4) of Theorem 1.1 can be complemented with the following (exact controllability) surjectivity result, arising this time from the continuous observability inequality $(1.15)$ by transposition or duality $[11,12]$.

THEOREM 1.4. With reference to the mixed problem (1.1), the map

$$
\left\{w_{0}, w_{1}\right\}=0, \quad u \in L_{2}\left(0, T ; L_{2}\left(\Gamma\left(x_{0}\right)\right)\right) \longrightarrow\left\{w(T), w_{t}(T)\right\}
$$

is surjective (onto) $H_{0}^{1}(\Omega) \times \tilde{L}_{2}(\Omega)$, for all $T>T_{0}>0$, with $T_{0}$ defined in (1.15). 
To further elaborate, any (target) state $\left\{v_{1}, v_{2}\right\}$ with $v_{1} \in H_{0}^{1}(\Omega)$ and $v_{2} \in \mathscr{H}$ (the null space of generalized harmonic functions defined in (2.5) below) cannot be reached from the origin over a time interval $[0, T], T>T_{0}$, by using an $L_{2}\left(0, T ; L_{2}\left(\Gamma\left(x_{0}\right)\right)\right)$-control. Similarly, by reversing time, an initial condition $\left\{w_{0}, w_{1}\right\}$ with $w_{0} \in H_{0}^{1}(\Omega)$ and $w_{1} \in \mathcal{H}$ cannot be steered to rest $(0,0)$ over the time interval $[0, T]$, by using an $L_{2}\left(0, T ; L_{2}\left(\Gamma\left(x_{0}\right)\right)\right)$-control. This is so since, by Proposition 2.7, any element $h \in \mathcal{H}$ has zero norm in $\tilde{L}_{2}(\Omega):\|h\|_{\tilde{L}_{2}(\Omega)}=0$, as the null space $\mathscr{H}$ of the operator $(1-\gamma \Delta)$ consisting of generalized harmonic functions defined in $(2.5)$ acts as the zero element in $\tilde{L}_{2}(\Omega)$.

In conclusion, Theorem 1.1 for $\left\{w, w_{t}\right\}$, as well as Theorem 1.4 do add new critical insight over the literature [7, 9]. In addition, the regularity (1.5) for $w_{t t}$ is entirely new. All this has critical implications on coupled systems such as thermoelastic systems, as well as viscoelastic systems [9].

Kirchhoff thermoelastic problem (1.2). For brevity, we limit our comments to the following considerations. The regularity (1.6) for $\left\{w, w_{t}\right\}$ is the same as that given (and proved) in [22]. However, regarding the regularity of $w_{t t}$ and $\theta$, the statements in (1.7), (1.8), and (1.9) of Theorem 1.2, as well as Theorem 1.3 represent a clarification over the literature [3, 4, 7, 22].

As the spaces $\tilde{L}_{2}(\Omega)$ and $\tilde{H}_{-1}(\Omega)$ (their definitions, their properties, such as (2.49), (3.19), and (3.38), and surrounding considerations) are not present in [7], we are unable to justify the claims for $w_{t}$ asserted to be in $L_{\infty}\left(0, T ; L_{2}(\Omega)\right)$, and for $\theta$ asserted to be in $L_{\infty}\left(0, T ; L_{2}(\Omega)\right)$ continuously in $u \in L_{2}\left(0, T ; L_{2}(\Gamma)\right)$, which are made in [7, equations (3.30), (3.31), page 160]: this reference states that they simply follow by a duality or transposition argument (such as the one from (1.17) to (1.24), in the elastic case) over the trace inequality in [7, equation (3.11), page 157], which is the counterpart of (1.14) in the thermoelastic case. (Even if $w_{t}$ were in $L_{\infty}\left(0, T ; L_{2}(\Omega)\right)$, then $\int_{0}^{t} e^{-\mathscr{A}(t-\tau)} \Delta w_{t}(\tau) d \tau$ would not be in $C\left([0, T] ; L_{2}(\Omega)\right)$, but only in $L_{p}\left(0, T ; L_{2}(\Omega)\right) \cap C\left([0, T] ; H^{-\epsilon}(\Omega)\right)$, for any $1<p<\infty$, and any $\epsilon>0$. There is no "maximal regularity" for the $L_{\infty}(0, T ; \cdot)$-spaces. See $(5.20)$ below. Moreover, our claim in this paper is that $w_{t} \in C\left([0, T] ; \tilde{L}_{2}(\Omega)\right)$ instead, as in (1.7).) By contrast we find that the regularity of $\theta \in C\left([0, T] ; L_{2}(\Omega)\right)$ is not continuous in $u \in L_{2}\left(0, T ; L_{2}(\Gamma)\right)$, see (1.9), and requires the analysis of $w_{t t}$, which involves the new space $\tilde{H}_{-1}(\Omega)$. To get continuity in $u \in L_{2}\left(0, T ; L_{2}(\Gamma)\right)$, lower topologies are involved in our analysis for $\theta$, as in (1.8) or (1.10) and (1.11). Theorem 1.3 requires a delicate trace analysis, which is sketched in Section 5.2.

Theorems 1.1, 1.2, and 1.3 are the main results of this paper regarding the (optimal at least for $\left\{w, w_{t}\right\}$ ) interior regularity of elastic and thermoelastic mixed problems, with clamped boundary controls. To achieve them, we need to introduce, and study the properties of two untraditional or new space $\tilde{L}_{2}(\Omega)$ and $\tilde{H}_{-1}(\Omega)$, below. These spaces occur also in describing the regularity of, say, the Kirchhoff elastic problem under irregular right-hand side. This is carried out 
in Section 4.2, which complements results in [21, Proposition 3.4], which were motivated by point control problems.

\section{The space $\tilde{L}_{2}(\Omega)$ and its properties}

We first introduce the operators which play a key role in the definition of the space $\tilde{L}_{2}(\Omega)$; and next we study their relevant properties.

2.1. The operators $A, A, A_{\gamma}$. The operator $A^{1 / 2} \mathscr{A}_{\gamma}^{-1}$. Let $\Omega$ be an open bounded domain in $\mathbb{R}^{n}$ with smooth boundary $\Gamma$. We define

$$
\begin{gathered}
A f=\Delta^{2} f, \quad \mathscr{D}(A)=H^{4}(\Omega) \cap H_{0}^{2}(\Omega) ; \\
\mathscr{A} f=-\Delta f, \quad \mathscr{A}_{\gamma}=I+\gamma \mathscr{A} ; \quad \mathscr{D}\left(\mathscr{A}_{\gamma}\right)=\mathscr{D}(\mathscr{A})=H^{2}(\Omega) \cap H_{0}^{1}(\Omega),
\end{gathered}
$$

so that, with equivalent norms, we have the following identifications:

$$
\mathscr{D}\left(A^{1 / 2}\right)=H_{0}^{2}(\Omega) ; \quad \mathscr{D}\left(A^{1 / 4}\right)=\mathscr{D}\left(\mathscr{A}^{1 / 2}\right)=\mathscr{D}\left(\mathscr{A}_{\gamma}^{1 / 2}\right)=H_{0}^{1}(\Omega) .
$$

The space $\mathscr{D}\left(\mathscr{A}_{\gamma}^{1 / 2}\right)$ will always be endowed with the following inner product, unless specifically noted otherwise:

$$
\begin{aligned}
\left(f_{1}, f_{2}\right)_{\mathscr{D}\left(\mathscr{A}_{\gamma}^{1 / 2}\right)} & =\left(\mathscr{A}_{\gamma}^{1 / 2} f_{1}, \mathscr{A}_{\gamma}^{1 / 2} f_{2}\right)_{L_{2}(\Omega)} \\
& =\left(\mathscr{A}_{\gamma} f_{1}, f_{2}\right)_{L_{2}(\Omega)}, \quad \forall f_{1}, f_{2} \in H_{0}^{1}(\Omega),
\end{aligned}
$$

where, at this stage, we denote with the same symbol the $L_{2}(\Omega)$-inner product and the duality pairing $(\cdot, \cdot)_{V^{\prime} \times V}, V=H_{0}^{1}(\Omega), V^{\prime}=H^{-1}(\Omega)$ with $L_{2}(\Omega)$ as a pivot space [1, Theorem 1.5, page 51], for the last term in (2.4).

The following closed subspaces of $L_{2}(\Omega)$ play a critical role. Consider the null space $\mathcal{N}$ of the operator $(1-\gamma \Delta): L_{2}(\Omega) \rightarrow H^{-2}(\Omega)=\left[\mathscr{D}\left(A^{1 / 2}\right)\right]^{\prime}$, and so let

$$
\mathscr{H} \equiv\left\{h \in L_{2}(\Omega):(1-\gamma \Delta) h=0 \text { in } H^{-2}(\Omega)\right\}=\mathcal{N}\{(1-\gamma \Delta)\}
$$

be the space of "generalized harmonic functions" in $L_{2}(\Omega)$. $\mathscr{H}$ depends on $\gamma$, of course. For instance, for $n=1$, we have $\mathscr{H}=\operatorname{span}\left\{e^{-\sqrt{(1 / \gamma) x}}, e^{\sqrt{(1 / \gamma) x}}\right\}$. Let $\mathcal{H}^{\perp}$ be its orthogonal complement in $L_{2}(\Omega)$ and $\Pi=\Pi^{*}$ be the orthogonal projection $L_{2}(\Omega)$ onto $\mathscr{H}^{\perp}$ :

$$
\begin{gathered}
\mathscr{H}^{\perp}=\left\{f \in L_{2}(\Omega):(f, h)_{L_{2}(\Omega)}=0, \forall h \in \mathscr{H}\right\} ; \\
L_{2}(\Omega)=\mathscr{H} \oplus \mathscr{H}^{\perp}, \quad \Pi L_{2}(\Omega)=\mathscr{H}^{\perp} .
\end{gathered}
$$

We start with an elementary lemma.

LEMMA 2.1. With reference to (2.1) and (2.2), 
(i)

$$
\begin{gathered}
\mathscr{A}_{\gamma} A^{-1 / 2} \in \mathscr{L}\left(L_{2}(\Omega)\right), \text { so that, by } L_{2}(\Omega) \text {-adjointness, } \\
A^{-1 / 2} \mathscr{A}_{\gamma} \text { has a bounded extension on } L_{2}(\Omega) .
\end{gathered}
$$

(ii) The subspace $\mathscr{H}$ in (2.5) is precisely the null space of the bounded operator $A^{-1 / 2} A_{\gamma}$ on $L_{2}(\Omega)$ :

$$
\begin{gathered}
\mathcal{N}\left\{A^{-1 / 2} \mathscr{A}_{\gamma}\right\}=\mathscr{H} ; \text { for } h \in \mathscr{H} \Longrightarrow A^{-1 / 2} \mathscr{A}_{\gamma} h=0 ; \text { and conversely, } \\
A^{-1 / 2} \mathscr{A}_{\gamma} h=0, \quad h \in L_{2}(\Omega) \Longrightarrow h \in \mathscr{H} .
\end{gathered}
$$

(Refer also to the subsequent (2.33).)

Proof. (i) By (2.2) and (2.3), we have that $\mathscr{D}\left(A^{1 / 2}\right) \subset \mathscr{D}(\mathscr{A})$, and then the closed graph theorem yields that $\mathscr{A}_{\gamma} A^{-1 / 2}$ is a bounded operator on $L_{2}(\Omega)$.

(ii) Here, and frequently below, we will use the second Green's identity

$$
(\Delta f, \phi)_{L_{2}(\Omega)}=(f, \Delta \phi)_{L_{2}(\Omega)}+\int_{\Gamma} \frac{\partial f}{\partial v} \phi d \Gamma-\int_{\Gamma} f \frac{\partial \phi}{\partial v} d \Gamma,
$$

whenever it makes sense. In particular, if $\phi \in H_{0}^{2}(\Omega)$, we can extend the validity of (2.9) to all $f \in L_{2}(\Omega)$, and write

$$
((1-\gamma \Delta) f, \phi)_{L_{2}(\Omega)}=(f,(1-\gamma \Delta) \phi)_{L_{2}(\Omega)}, \quad f \in L_{2}(\Omega), \phi \in H_{0}^{2}(\Omega) .
$$

Let now $f \in L_{2}(\Omega)$ so that $\phi \equiv A^{-1 / 2} f \in H_{0}^{2}(\Omega) \subset \mathscr{D}\left(\mathscr{A}_{\gamma}\right)$ by $(2.3)$ and (2.2). Take $h \in L_{2}(\Omega)$, so that $A^{-1 / 2} \mathscr{A}_{\gamma} h \in L_{2}(\Omega)$ is well defined by (2.7). Then, via identity (2.10), we obtain

$$
\begin{aligned}
\left(A^{-1 / 2} \mathscr{A}_{\gamma} h, f\right)_{L_{2}(\Omega)} & =\left(\mathscr{A}_{\gamma} h, A^{-1 / 2} f\right)_{L_{2}(\Omega)}=\left(h, \mathscr{A}_{\gamma} \phi\right)_{L_{2}(\Omega)} \\
& =(h,(1-\gamma \Delta) \phi)_{L_{2}(\Omega)}=((1-\gamma \Delta) h, \phi)_{L_{2}(\Omega)} .
\end{aligned}
$$

Now, if $h \in \mathscr{H}$, then, by (2.5), the right-hand side of (2.11) is zero for all $f \in L_{2}(\Omega)$ and so, by the left-hand side, $A^{-1 / 2} A_{\gamma} h=0$, as desired. Conversely, if $A^{-1 / 2} A_{\gamma} h=0$, then the right-hand side of (2.11) implies that $(1-\gamma \Delta) h=0$ in $H^{-2}(\Omega)$, and thus $h \in \mathscr{H}$ by (2.5).

Lemma 2.1(ii) says that $\mathscr{H}$ is precisely the "invisible" subspace of the operator $A^{-1 / 2} A_{\gamma} \in \mathscr{L}\left(L_{2}(\Omega)\right)$. The following lemma lists critical properties of the operator $A^{1 / 2} \mathscr{A}_{\gamma}^{-1}$.

Lemma 2.2. With reference to (2.1), (2.2), and (2.6), 
Factor spaces and Kirchhoff equations

(a1)

$$
\begin{gathered}
\mathscr{A}_{\gamma}: \text { continuous } H_{0}^{2}(\Omega) \equiv \mathscr{D}\left(A^{1 / 2}\right) \longrightarrow \mathscr{H}^{\perp} ; \\
\text { equivalently, } \mathscr{A}_{\gamma} A^{-1 / 2}: \text { continuous } L_{2}(\Omega) \longrightarrow \mathscr{H}^{\perp},
\end{gathered}
$$

thus improving upon Lemma 2.1(i), (2.7).

(a2) $A_{\gamma} A^{-1 / 2}$ is injective (one-to-one) on $L_{2}(\Omega)$

$$
\mathscr{A}_{\gamma} A^{-1 / 2} x=0, \quad x \in L_{2}(\Omega) \Longrightarrow x=0 .
$$

(a3) For $F \in L_{2}(\Omega)$,

$$
\left.f \equiv \mathscr{A}_{\gamma}^{-1} F \in \mathscr{D}\left(A^{1 / 2}\right) \Longleftrightarrow \frac{\partial f}{\partial v}\right|_{\Gamma}=\left.\frac{\partial \mathscr{A}_{\gamma}^{-1} F}{\partial v}\right|_{\Gamma}=0 \Longleftrightarrow F \in \mathscr{H}^{\perp} ;
$$

equivalently, $A^{1 / 2} A_{\gamma}^{-1}$, as an operator on $L_{2}(\Omega)$, has a domain given precisely by $\mathscr{H}^{\perp}: \mathscr{D}\left(A^{1 / 2} \mathscr{A}_{\gamma}^{-1}\right)=\mathscr{H}^{\perp}$, in which case $A^{1 / 2} \mathscr{A}_{\gamma}^{-1}:$ continuous $\mathscr{H}^{\perp} \longrightarrow L_{2}(\Omega)$.

(a4) The above maps in (2.12) are, in fact, surjective:

$$
\mathscr{A}_{\gamma} \text { : continuous from } H_{0}^{2}(\Omega) \equiv \mathscr{D}\left(A^{1 / 2}\right) \text { onto } \mathscr{H}^{\perp} \text {; }
$$

equivalently, $\mathscr{A}_{\gamma} A^{-1 / 2}$ : continuous from $L_{2}(\Omega)$ onto $\mathscr{H}^{\perp}$. (a5)

$$
\mathscr{A}_{\gamma} A^{-1 / 2} \text { is an isomorphism from } L_{2}(\Omega) \text { onto } \mathcal{H}^{\perp},
$$

with bounded inverse

$$
\begin{gathered}
\left(\mathscr{A}_{\gamma} A^{-1 / 2}\right)^{-1}=A^{1 / 2} \mathscr{A}_{\gamma}^{-1} \text { continuous from } \mathscr{H}^{\perp} \text { onto } L_{2}(\Omega) ; \\
\text { equivalently, recalling } \Pi L_{2}(\Omega)=\mathscr{H}^{\perp} \text { from }(2.6) \\
A^{1 / 2} \mathscr{A}_{\gamma}^{-1} \Pi: \text { continuous } L_{2}(\Omega) \text { onto } L_{2}(\Omega),
\end{gathered}
$$

and by $L_{2}(\Omega)$-adjointness

$$
\Pi \mathscr{A}_{\gamma}^{-1} A^{1 / 2} \text { has a continuous extension } L_{2}(\Omega) \longrightarrow L_{2}(\Omega),
$$

where $\Pi=\Pi^{*}$ is the orthogonal projection from $L_{2}(\Omega)$ onto $\mathscr{H}^{\perp}$.

(b) Complementing (a3), for $0 \neq h \in \mathscr{H} \subset L_{2}(\Omega)$, then

$$
\psi=\mathscr{A}_{\gamma}^{-1} h \in \mathscr{D}\left(\mathscr{A}_{\gamma}\right)=H^{2}(\Omega) \cap H_{0}^{1}(\Omega)
$$

does not belong to $\mathscr{D}\left(A^{1 / 2}\right)$, since $\left.\frac{\partial \psi}{\partial v}\right|_{\Gamma} \neq 0$; and, in fact,

$$
-\gamma \int_{\Gamma} \frac{\partial \psi}{\partial v} h d \Gamma=\|h\|_{L_{2}(\Omega)}^{2}, \quad \forall h \in \mathscr{H} .
$$


Proof. (a1) Let $f \in H_{0}^{2}(\Omega) \subset \mathscr{D}\left(\mathscr{A}_{\gamma}\right)$, and define $F \equiv \mathscr{A}_{\gamma} f \in L_{2}(\Omega)$. To show (a1), we compute by Green's identity (2.10) and (2.5):

$$
\begin{aligned}
(F, h)_{L_{2}(\Omega)} & =\left(\mathscr{A}_{\gamma} f, h\right)=((1-\gamma \Delta) f, h)_{L_{2}(\Omega)} \\
& =(f,(1-\gamma \Delta) h)_{L_{2}(\Omega)}=0, \quad \forall h \in \mathscr{H},
\end{aligned}
$$

and thus $F=\mathscr{A}_{\gamma} f \in \mathscr{H}^{\perp}$, for all $f \in H_{0}^{2}(\Omega)$, as desired. Then, (a2) is immediate, since $A^{-1 / 2} x \in \mathscr{D}\left(\mathscr{A}_{\gamma}\right)$ for $x \in L_{2}(\Omega)$.

(a3) Let $F \in L_{2}(\Omega)$ and define $f \equiv \mathscr{A}_{\gamma}^{-1} F \in \mathscr{D}\left(\mathscr{A}_{\gamma}\right)=H^{2}(\Omega) \cap H_{0}^{1}(\Omega)$ so that $\left.f\right|_{\Gamma}=0$. Then, $f \in H_{0}^{2}(\Omega)=\mathscr{D}\left(A^{1 / 2}\right)$ if and only if, in addition, $\left.(\partial f / \partial v)\right|_{\Gamma}=0$, and the first equivalence in (2.14a) is established. Next, take $h \in \mathscr{H}$ and compute by Green's identity (2.9) and (2.5), with $F=\mathscr{A}_{\gamma} f=$ $(1-\gamma \Delta) f,\left.f\right|_{\Gamma}=0,\left.(\partial f / \partial v)\right|_{\Gamma} \in H^{1 / 2}(\Gamma)$ :

$$
\begin{aligned}
(F, h)_{L_{2}(\Omega)} & =((1-\gamma \Delta) f, h)_{L_{2}(\Omega)} \\
& =(f,(1-\gamma \Delta) h)_{L_{2}(\Omega)}-\gamma \int_{\Gamma} \frac{\partial f}{\partial v} h d \Gamma \\
& =-\gamma \int_{\Gamma} \frac{\partial f}{\partial v} h d \Gamma, \quad \forall h \in \mathcal{H} .
\end{aligned}
$$

Now if $f \in \mathscr{D}\left(A^{1 / 2}\right)$, equivalently if $\left.(\partial f / \partial v)\right|_{\Gamma}=0$ as seen above, then (2.23) yields $(F, h)_{L_{2}(\Omega)}=0$ for all $h \in \mathscr{H}$, and then $F \in \mathscr{H}^{\perp}$.

Conversely, if $F \in \mathscr{H}^{\perp}$, then (2.23) yields $\int_{\Gamma}(\partial f / \partial \nu) h d \Gamma=0$, for all $h \in \mathscr{H}$.

We next show that this then implies that $\left.(\partial f / \partial v)\right|_{\Gamma}=0$, as desired. In fact, for any $u \in H^{-1 / 2}(\Gamma)$, define the Dirichlet map $D_{\gamma}$ by

$$
D_{\gamma} u=h \Longleftrightarrow\left\{\begin{array}{ll}
(1-\gamma \Delta) h=0 & \text { in } \Omega, \\
h=u & \text { in } \Gamma,
\end{array} \quad u \in H^{-1 / 2}(\Gamma),\right.
$$

so that the well-posed elliptic problem produces a unique solution $h=D_{\gamma} u \in$ $L_{2}(\Omega)$ and then $h \in \mathscr{H}$ by (2.5). Thus, the traces $\left.h\right|_{\Gamma}$ of such solutions fill all of $H^{-1 / 2}(\Gamma)$ and then the established identity $\int_{\Gamma}(\partial f / \partial v) h d \Gamma=0$ with $\left.(\partial f / \partial v)\right|_{\Gamma} \in H^{1 / 2}(\Gamma)$ implies $\left.(\partial f / \partial v)\right|_{\Gamma}=0$, as desired.

Continuity of $A^{1 / 2} A_{\gamma}^{-1}$ as an operator: $\mathscr{H}^{\perp} \rightarrow L_{2}(\Omega)$ follows now by the closed graph theorem.

(a4) The surjectivity property in (a4) follows at once from the established property (a3). Let $F \in \mathscr{H}^{\perp}$ and define $\phi=A^{1 / 2} \mathscr{A}_{\gamma}^{-1} F \in L_{2}(\Omega)$, which is well defined by (a3). Then $\mathscr{A}_{\gamma} A^{-1 / 2} \phi=F \in \mathscr{H}^{\perp}$ and the continuous injective operator $\mathscr{A}_{\gamma} A^{-1 / 2}$ is surjective from $L_{2}(\Omega)$ onto $\mathscr{H}^{\perp}$.

(a5) The bounded, injective, surjective operator $\mathscr{A}_{\gamma} A^{-1 / 2}$ from $L_{2}(\Omega)$ onto $\mathscr{H}^{\perp}$ (recall (2.12), (2.13), and (2.15)) has, by virtue of the open mapping theorem, a continuous inverse $\left(\mathscr{A}_{\gamma} A^{-1 / 2}\right)^{-1}$, which is then given by (2.17). 
(b) Let now $h \in \mathscr{H} \subset L_{2}(\Omega)$ and define $\psi \equiv \mathscr{A}_{\gamma}^{-1} h \in \mathscr{D}\left(\mathscr{A}_{\gamma}\right)=H^{2}(\Omega) \cap$ $H_{0}^{1}(\Omega)$ so that $\mathscr{A}_{\gamma} \psi=h$ and

$$
(1-\gamma \Delta) \psi=h \quad \text { in } \Omega ;\left.\quad \psi\right|_{\Gamma}=0 .
$$

Taking the inner product in (2.25) with $h$, and invoking once more the Green's identity (2.9), we obtain (as in (2.22))

$$
((1-\gamma \Delta) \psi, h)_{L_{2}(\Omega)}=(h, h)_{L_{2}(\Omega)}=(\psi,(1-\gamma \Delta) h)_{L_{2}(\Omega)}-\gamma \int_{\Gamma} \frac{\partial \psi}{\partial v} h d \Gamma,
$$

or, by (2.5), we see that (2.20) follows from (2.26) for all $h \in \mathcal{H}$. Then (2.20) implies that $\left.(\partial \psi / \partial v)\right|_{\Gamma} \neq 0$ for $h \neq 0$.

2.2. Definition of the space $\tilde{L}_{2}(\Omega)$. Equivalent formulations. The definition of the following space arises in duality considerations involving Kirchhoff elastic problems with clamped boundary conditions and their corresponding thermoelastic versions. This was already explained in the PDE duality analysis, beginning with (1.17) and leading to (1.24). This will also be explained in Section 4, see the critical (4.12) and (4.66), in a systematic functional analytic approach. We consider (see (2.1), (2.2), (2.3), and (2.4)):

(i) the space $\mathscr{D}\left(A^{1 / 2}\right) \equiv H_{0}^{2}(\Omega)$ as a closed subspace of

$$
\mathscr{D}\left(\mathscr{A}_{\gamma}\right) \equiv H^{2}(\Omega) \cap H_{0}^{1}(\Omega) ;
$$

(ii) the space $\mathscr{D}\left(\mathscr{A}_{\gamma}^{1 / 2}\right)$ as a pivot space, with norm as in (2.4),

$$
\|f\|_{\mathscr{D}\left(\mathscr{A}_{\gamma}^{1 / 2}\right)}^{2}=\left(\mathscr{A}_{\gamma}^{1 / 2} f, \mathscr{A}_{\gamma}^{1 / 2} f\right)_{L_{2}(\Omega)}, \quad \forall f \in \mathscr{D}\left(\mathscr{A}_{\gamma}^{1 / 2}\right) \equiv H_{0}^{1}(\Omega) .
$$

The space $\mathscr{D}\left(A^{1 / 2}\right)$ is dense in $\mathscr{D}\left(\mathscr{A}_{\gamma}^{1 / 2}\right)$, however, so the identification result $\mathscr{D}\left(A^{1 / 2}\right) \subset \mathscr{D}\left(\mathscr{A}_{\gamma}^{1 / 2}\right) \subset\left[\mathscr{D}\left(A^{1 / 2}\right)\right]^{\prime}$ in [1, page 51] applies with duality with respect to $\mathscr{D}\left(\mathscr{A}_{\gamma}^{1 / 2}\right)$ as a pivot space. We then define the (Hilbert) space $\tilde{L}_{2}(\Omega)$ as follows:

$$
\begin{aligned}
\tilde{L}_{2}(\Omega)= & \text { dual of the space } \mathscr{D}\left(A^{1 / 2}\right) \text { with respect to the space } \mathscr{D}\left(\mathscr{A}_{\gamma}^{1 / 2}\right) \\
& \text { as a pivot space, endowed with the norm of }(2.28)(\text { or }(2.4)) .
\end{aligned}
$$

This means the following: let $f \in \mathscr{D}\left(A^{1 / 2}\right) \equiv H_{0}^{2}(\Omega) \subset \mathscr{D}\left(\mathscr{A}_{\gamma}\right)$, or $\phi=A^{1 / 2} f \in$ $L_{2}(\Omega)$. Then

$$
\begin{aligned}
g \in \tilde{L}_{2}(\Omega) \Longleftrightarrow(f, g)_{\mathscr{D}\left(\mathscr{A}_{\gamma}^{1 / 2}\right)} & =\left(\mathscr{A}_{\gamma} f, g\right)_{L_{2}(\Omega)}=\text { finite, } \quad \forall f \in H_{0}^{2}(\Omega) \\
& =\left(f, \mathscr{A}_{\gamma} g\right)_{L_{2}(\Omega)}=\left(A^{-1 / 2} \phi, \mathscr{A}_{\gamma} g\right)_{L_{2}(\Omega)} \\
& =\left(\phi, A^{-1 / 2} \mathscr{A}_{\gamma} g\right)_{L_{2}(\Omega)}=\text { finite, } \quad \forall \phi \in L_{2}(\Omega),
\end{aligned}
$$


where we write in the same way inner products and corresponding duality pairings.

Proposition 2.3. (i) Definition (2.30) is equivalent to the following restatement:

$$
\begin{aligned}
g \in \tilde{L}_{2}(\Omega) \Longleftrightarrow\left(\mathscr{A}_{\gamma} f, g\right)_{L_{2}(\Omega)} & =(F, g)_{L_{2}(\Omega)} \\
& =((1-\gamma \Delta) f, g)_{L_{2}(\Omega)} \\
& =(f,(1-\gamma \Delta) g)_{L_{2}(\Omega)}=\text { finite }
\end{aligned}
$$

for all $f \in H_{0}^{2}(\Omega)$, or for all $F \in \mathscr{H}^{\perp}$, where $F=\mathscr{A}_{\gamma} f=(1-\gamma \Delta) f$.

(ii) Definition (2.30) is equivalent to the following restatement:

$$
g \in \tilde{L}_{2}(\Omega) \Longleftrightarrow A^{-1 / 2} A_{\gamma} g \in L_{2}(\Omega) .
$$

Refer also to Lemma 2.1(ii), (2.8) that $\mathcal{N}\left(A^{-1 / 2} \mathscr{A}_{\gamma}\right)=\mathscr{H}$.

(iii) The following set-theoretic and algebraic (but not topological, see Proposition 2.7 below for the topological statement, (2.49)) inclusion $\tilde{L}_{2}(\Omega) \supset$ $L_{2}(\Omega)$ holds.

Proof. (i) By Lemma 2.2(a4), (2.15), we know that the range $\mathscr{A}_{\gamma}\left[H_{0}^{2}(\Omega)\right]$ of all $H_{0}^{2}(\Omega)=\mathscr{D}\left(A^{1 / 2}\right)$ under the action of $\mathscr{A}_{\gamma}$ is precisely the subspace $\mathscr{H}^{\perp}$ in (2.6). This yields the first equality in (2.31). Once $\mathscr{A}_{\gamma} f=(1-\gamma \Delta) f$ is used for $f \in H_{0}^{2}(\Omega)$, then the remaining equality in (2.32) follows from Green's identity (2.10).

(ii) Part (ii), (2.33), follows at once from (2.30b).

(iii) Clearly any element $g \in \mathscr{H}^{\perp}$ or $g \in \mathscr{H}$ makes (2.31) finite, and so part (iii) follows as a set-theoretic inclusion.

Remark 2.4. We will see after Proposition 2.7 that $\tilde{L}_{2}(\Omega)$ coincides with $\mathscr{H}^{\perp}$ topologically, and with $L_{2}(\Omega)$ set-theoretically, also by part (iii) above.

2.3. Further description of the space $\tilde{L}_{2}(\Omega)$. Lemma $2.2(\mathrm{a} 4)$ has permitted us to rewrite the original definition (2.30) in the equivalent, and more descriptive, form (2.31). Taking the latter as our starting point, we have the following proposition.

Proposition 2.5. (a) With reference to (2.31), $g \in \tilde{L}_{2}(\Omega) \Longleftrightarrow\left\{\begin{array}{l}g \text { has a component } g_{1} \text { defined by } g_{1}=\Pi g=\left.g\right|_{\mathscr{H}} ^{\perp} \in \mathscr{H}^{\perp} \subset L_{2}(\Omega) \\ \text { which is the orthogonal projection of } g \text { onto } \mathscr{H}^{\perp},\end{array}\right.$ in which case

(i)

$$
(1-\gamma \Delta) g=(1-\gamma \Delta) g_{1} \quad \text { in } H^{-2}(\Omega)
$$


454 Factor spaces and Kirchhoff equations

(ii)

$$
A^{-1 / 2} \mathscr{A}_{\gamma} g=A^{-1 / 2} \mathscr{A}_{\gamma} g_{1} \in L_{2}(\Omega) .
$$

Recall also Lemma 2.1(ii): $\mathcal{N}\left(A^{-1 / 2} \mathscr{A}_{\gamma}\right)=\mathscr{H}$.

(b) Let $g \in \tilde{L}_{2}(\Omega)$. Its norm is

$$
\|g\|_{\tilde{L}_{2}(\Omega)}=\sup _{F \in \mathscr{H}^{\perp} ;\|F\|_{L_{2}(\Omega)}=1}\left\{\left|\left(F, g_{1}\right)_{L_{2}(\Omega)}\right|\right\}=\left\|g_{1}\right\|_{L_{2}(\Omega)} .
$$

In particular,

$$
\|h\|_{\tilde{L}_{2}(\Omega)}=0, \quad \forall h \in \mathscr{H} ; \quad\|F\|_{\tilde{L}_{2}(\Omega)}=\|F\|_{L_{2}(\Omega)}, \quad \forall F \in \mathscr{H}^{\perp} .
$$

Proof. First, from definition (2.31), $(F, g)_{L_{2}(\Omega)}$ is finite, for all $F \in \mathscr{H}^{\perp}$ implies property (2.34). Next, by part (2.34) and (2.15a), we can rewrite (2.31) for $f \in H_{0}^{2}(\Omega)$ as

$$
\left(\mathscr{A}_{\gamma} f, g\right)_{L_{2}(\Omega)}=\left(\mathscr{A}_{\gamma} f, g_{1}\right)_{L_{2}(\Omega)},
$$

or setting $f=A^{-1 / 2} \phi \in \mathscr{D}\left(A^{1 / 2}\right)=H_{0}^{2}(\Omega)$, for $\phi \in L_{2}(\Omega)$,

$$
\left(\phi, A^{-1 / 2} \mathscr{A}_{\gamma} g\right)_{L_{2}(\Omega)}=\left(\phi, A^{-1 / 2} \mathscr{A}_{\gamma} g_{1}\right)_{L_{2}(\Omega)}, \quad \forall \phi \in L_{2}(\Omega),
$$

from which (2.36) follows. Next, we rewrite (2.38a) explicitly (as in (2.31) and (2.32)) as follows via Green's identity (2.10)

$$
\begin{aligned}
(f,(1-\gamma \Delta) g)_{L_{2}(\Omega)} & =((1-\gamma \Delta) f, g)_{L_{2}(\Omega)}=\left(\mathscr{A}_{\gamma} f, g\right)_{L_{2}(\Omega)} \\
& =\left(\mathscr{A}_{\gamma} f, g_{1}\right)_{L_{2}(\Omega)}=\left((1-\gamma \Delta) f, g_{1}\right)_{L_{2}(\Omega)} \\
& =\left(f,(1-\gamma \Delta) g_{1}\right)_{L_{2}(\Omega)}, \quad \forall f \in H_{0}^{2}(\Omega) .
\end{aligned}
$$

Then the first and the last term in identity (2.39) yield property (i) in (2.35), as desired. Similarly, from (2.38b), we get part (ii) in (2.36). Part (b) is a selfexplanatory consequence of part (a).

Proposition 2.6. (i) For $g_{1}$ defined in (2.34), (2.35), and (2.36)

$$
G_{1}=A^{-1 / 2} \mathscr{A}_{\gamma} g_{1} \in L_{2}(\Omega) \text {. }
$$

(ii) The operator $\left[A^{-1 / 2} \mathscr{A}_{\gamma}\right]_{\mathscr{H}} \perp$ (= restriction of the continuous operator $\left(A^{-1 / 2} \mathscr{A}_{\gamma}\right)$ to $\left.\mathscr{H}^{\perp}\right)$ is injective as an operator $\mathscr{H}^{\perp} \rightarrow L_{2}(\Omega)$

$$
\left[A^{-1 / 2} \mathscr{A}_{\gamma}\right]_{\mathscr{H}^{\perp}} x=0, \quad x \in \mathscr{H}^{\perp} \Longrightarrow x=0 .
$$

This is a restatement of Lemma 2.1(ii), (2.8), $\mathcal{N}\left(A^{-1 / 2} \mathscr{A}_{\gamma}\right)=\mathscr{H}$. 
(iii) The components $g_{1}=\Pi g$ of all $g \in \tilde{L}_{2}(\Omega)$ are given as

$$
g_{1}=\left(\left[A^{-1 / 2} \mathscr{A}_{\gamma}\right]_{\mathscr{H}^{\perp}}\right)^{-1} G_{1}, \quad \forall G_{1} \in L_{2}(\Omega),
$$

or

$$
\mathscr{H}^{\perp}=\left(\left[A^{-1 / 2} \mathscr{A}_{\gamma}\right]_{\mathscr{H}^{\perp}}\right]^{-1} L_{2}(\Omega)=\Pi \mathscr{A}_{\gamma}^{-1} A^{1 / 2} L_{2}(\Omega),
$$

where

$$
\left(\left[A^{-1 / 2} \mathscr{A}_{\gamma}\right]_{\mathscr{H}^{\perp}}\right]^{-1}=\Pi \mathscr{A}_{\gamma}^{-1} A^{1 / 2}: \text { continuous } L_{2}(\Omega) \longrightarrow L_{2}(\Omega) .
$$

Proof. (i) By (2.7), $A^{-1 / 2} \mathscr{A}_{\gamma}$ has a bounded extension on $L_{2}(\Omega)$. Moreover, $g_{1} \in \mathscr{H}^{\perp} \subset L_{2}(\Omega)$. So (2.40) follows; see also (2.36).

(ii) With $x \in \mathscr{H}^{\perp}$, assume the left-hand side of (2.41). Then, for $f \in L_{2}(\Omega)$,

$$
\begin{aligned}
0 & =\left(\left[A^{-1 / 2} \mathscr{A}_{\gamma}\right]_{\mathscr{H}^{\perp}} x, f\right)_{L_{2}(\Omega)} \\
& =\left(A^{-1 / 2} \mathscr{A}_{\gamma} x, f\right)_{L_{2}(\Omega)}=\left(x, \mathscr{A}_{\gamma} A^{-1 / 2} f\right)_{L_{2}(\Omega)} .
\end{aligned}
$$

But by Lemma 2.2(a4), we have that $\mathscr{A}_{\gamma} A^{-1 / 2} L_{2}(\Omega)=\mathscr{H}^{\perp}$. Since $x \in \mathscr{H}^{\perp}$, then (2.44) implies that $x=0$, as desired. Recall also Lemma 2.1(ii).

(iii) Since $g_{1}=\Pi g=\left.g\right|_{\mathscr{H}} \perp$ by (2.5), then (2.40) can be rewritten as

$$
\left[A^{-1 / 2} \mathscr{A}_{\gamma}\right]_{\mathscr{H}^{\perp}} g_{1}=G_{1} \in L_{2}(\Omega) \text {, hence } g_{1}=\left(\left[A^{-1 / 2} \mathscr{A}_{\gamma}\right]_{\mathscr{H}^{\perp}}\right)^{-1} G_{1} \in \mathscr{H}^{\perp} \text {, }
$$

by invoking the injectivity of part (ii). It remains to establish identity (2.43), where continuity: $L_{2}(\Omega) \rightarrow$ itself, of its right-hand side was obtained in (2.18) of Lemma 2.2(a5). Let $x \in \mathscr{H}^{\perp}$ and set

$$
\left[A^{-1 / 2} \mathscr{A}_{\gamma}\right]_{\mathscr{H}^{\perp}} x=A^{-1 / 2} \mathscr{A}_{\gamma} x=y \in L_{2}(\Omega) .
$$

Then for any $f \in L_{2}(\Omega)$,

$$
\begin{aligned}
\left(x, \mathscr{A}_{\gamma} A^{-1 / 2} f\right)_{L_{2}(\Omega)} & =\left(A^{-1 / 2} \mathscr{A}_{\gamma} x, f\right)_{L_{2}(\Omega)}=(y, f)_{L_{2}(\Omega)} \\
& =\left(y, A^{1 / 2} \mathscr{A}_{\gamma}^{-1} \mathscr{A}_{\gamma} A^{-1 / 2} f\right)_{L_{2}(\Omega)} \\
& =\left(\mathscr{A}_{\gamma}^{-1} A^{1 / 2} y, \mathscr{A}_{\gamma} A^{-1 / 2} f\right)_{L_{2}(\Omega)}, \quad \forall f \in L_{2}(\Omega) .
\end{aligned}
$$

Since, by Lemma $2.2(\mathrm{a} 4), \mathscr{A}_{\gamma} A^{-1 / 2} L_{2}(\Omega)=\mathscr{H}^{\perp}$, then $(2.47)$ can be rewritten as

$$
\left(x, \mathscr{A}_{\gamma} A^{-1 / 2} f\right)_{L_{2}(\Omega)}=\left(\Pi \mathscr{A}_{\gamma}^{-1} A^{1 / 2} y, \mathscr{A}_{\gamma} A^{-1 / 2} f\right)_{L_{2}(\Omega)}, \quad \forall f \in L_{2}(\Omega),
$$

and then $x=\Pi \mathscr{A}_{\gamma}^{-1} A^{1 / 2} y$, as desired, and (2.43) follows also via (2.46), and (ii).

The above indicates that, with $\tilde{L}_{2}(\Omega)$, we are in the situation similar to that of a seminormed linear space: this, then, can be transformed into a normed space as a factor (quotient) space. That this is the case is shown next. 
2.4. The space $\tilde{L}_{2}(\Omega)$ is isometric to the factor space $L_{2}(\Omega) / \mathscr{H}$. Proposition 2.5 suggests that $\tilde{L}_{2}(\Omega)$ is isometric to the factor (or quotient) space $L_{2}(\Omega) / \mathscr{H}$, hence to $\mathscr{H}^{\perp}$ : in $\tilde{L}_{2}(\Omega)$, all generalized harmonic functions $h \in \mathscr{H}$ have zero $\tilde{L}_{2}(\Omega)$-norm. This result is correct and is given below.

Proposition 2.7. The space $\tilde{L}_{2}(\Omega)$ as defined in (2.29) is isometrically isomorphic (congruent, in the terminology of [20, page 53]) to the factor (or quotient) space $L_{2}(\Omega) / \mathscr{H}$, where $\mathscr{H}$ is defined by (2.5). In symbols:

$$
\tilde{L}_{2}(\Omega) \cong L_{2}(\Omega) / \mathscr{H} \cong \mathscr{H}^{\perp} \text {. }
$$

Thus, if $J$ denotes the isometric isomorphism between $\tilde{L}_{2}(\Omega)$ and $L_{2}(\Omega) / \mathscr{H}$, then for $g \in \tilde{L}_{2}(\Omega)$

$$
\|g\|_{\tilde{L}_{2}(\Omega)}=\|[J g]\|_{L_{2}(\Omega) / \mathscr{H}}=\inf _{h \in \mathscr{H}}\|J g-h\|_{L_{2}(\Omega)}=\left\|g_{1}\right\|_{L_{2}(\Omega)},
$$

for the unique element $g_{1}=\Pi g \in \mathscr{H}^{\perp}, g_{1} \in[\mathrm{Jg}]$ (the latter being the coset or equivalence class of $L_{2}(\Omega) / \mathscr{H}$ containing the element $J g$ )

$$
\begin{aligned}
(x, y)_{\tilde{L}_{2}(\Omega)} & =([J x],[J y])_{L_{2}(\Omega) / \mathscr{H}} \\
& =(\xi, \eta)_{L_{2}(\Omega)}=\left(x_{1}, y_{1}\right)_{L_{2}(\Omega)}, \quad \forall \xi \in[J x], \eta \in[J y],
\end{aligned}
$$

where $x_{1}=\Pi x, y_{1}=\Pi y$.

Proof. To prove (2.49), we will use a standard result [1, Theorem 1.6, page 53], [20, Theorem 3.5, page 135], and [5, Theorem 6.11, page 118]. Using Aubin's notation, we set

$$
P \equiv \mathscr{D}\left(A^{1 / 2}\right): \text { a closed subspace of } V \equiv \mathscr{D}\left(\mathscr{A}_{\gamma}\right)
$$

equipped with the inner product

$$
(w, v)_{V}=\left(\mathscr{A}_{\gamma} w, \mathscr{A}_{\gamma} v\right)_{L_{2}(\Omega)}
$$

By the above references, we have that

$P^{\prime}$ is isometrically isomorphic (congruent) to the factor space $V^{\prime} / P^{\perp}$,

where

$$
\begin{aligned}
P^{\perp} & \equiv\left\{f \in V^{\prime}: f(v)=(f, v)_{V^{\prime} \times V}=0, \forall v \in P \subset V\right\} ; \\
P^{\prime} & \equiv \text { space of continuous linear functionals on } P \\
V^{\prime} & \equiv \text { space of continuous linear functionals on } V
\end{aligned}
$$


and $(,)_{V^{\prime} \times V}$ denotes the duality pairing on $V^{\prime} \times V$. We now take

$$
\begin{aligned}
& \mathscr{D}\left(\mathscr{A}_{\gamma}^{1 / 2}\right) \text { (equipped with the norm as in (2.28)) } \\
& \text { as a common pivot space for } P \text { and } V,
\end{aligned}
$$

and we note that $P$ is dense in $\mathscr{D}\left(\mathscr{A}_{\gamma}^{1 / 2}\right)$. Then

(i) $P^{\prime}$ can be isometrically identified with the space, see definition (2.29),

$$
\begin{aligned}
\tilde{L}_{2}(\Omega) \equiv & \text { dual of }\left[\mathscr{D}\left(A^{1 / 2}\right) \equiv P\right] \\
& \text { with respect to } \mathscr{D}\left(\mathscr{A}_{\gamma}^{1 / 2}\right)=\left[\mathscr{D}\left(A^{1 / 2}\right)\right]_{\text {w.r.t. }}^{\prime} \mathscr{D}\left(\mathscr{A}_{\gamma}^{1 / 2}\right)^{\bullet}
\end{aligned}
$$

(ii) $V^{\prime}$ can be isometrically identified with the space

$$
L_{2}(\Omega) \equiv\left[\mathscr{D}\left(\mathscr{A}_{\gamma}\right)\right]_{\text {W.r.t. } \mathscr{D}\left(\mathscr{A}_{\gamma}^{1 / 2}\right)}^{\prime}
$$

Next, we find the corresponding isometric identification for $P^{\perp}$ (which is a closed subspace of $V^{\prime}$ ). By the Riesz representation theorem, if $I$ denotes the canonical isometry from $V$ onto $V^{\prime}$, then $P^{\perp}$ in (2.54) can be isometrically identified with the following subspace of $V$ :

$$
\begin{aligned}
& \left\{I^{-1} f \in V: \text { inner product }\left(I^{-1} f, v\right)_{V}=0, \forall v \in P \subset V\right\} \\
& \quad \equiv\left\{w \in V \equiv \mathscr{D}\left(\mathscr{A}_{\gamma}\right):(w, v)_{V}=\left(\mathscr{A}_{\gamma} w, \mathscr{A}_{\gamma} v\right)_{L_{2}(\Omega)}=0, \forall v \in P\right\} \\
& \quad=\left\{h \in L_{2}(\Omega):\left(h, \mathscr{A}_{\gamma} v\right)_{L_{2}(\Omega)}=0, \forall v \in P=\mathscr{D}\left(A^{1 / 2}\right) \equiv H_{0}^{2}(\Omega) \subset \mathscr{D}\left(\mathscr{A}_{\gamma}\right)\right\} \\
& \quad=\left\{h \in L_{2}(\Omega):(h,(1-\gamma \Delta) v)_{L_{2}(\Omega)}=0, \forall v \in P \equiv H_{0}^{2}(\Omega)\right\} \\
& \quad=\left\{h \in L_{2}(\Omega):((1-\gamma \Delta) h, v)_{L_{2}(\Omega)}=0, \forall v \in P \equiv H_{0}^{2}(\Omega)\right\} \\
& \quad=\left\{h \in L_{2}(\Omega):(1-\gamma \Delta) h=0, \text { in } H^{-2}(\Omega)\right\} \equiv \mathscr{H}=\mathcal{N}\{(1-\gamma \Delta)\},
\end{aligned}
$$

the null space of the operator $(1-\gamma \Delta): L_{2}(\Omega) \rightarrow H^{-2}(\Omega)$, introduced in (2.5). In going through the steps above, we have used: the inner product $(2.52 \mathrm{~b})$ from (2.60) to (2.61); the definition of $\mathscr{A}_{\gamma}$ given by (2.2) in (2.15); the Green's identity (2.10) from (2.15) to (2.16), since $v \in H_{0}^{2}(\Omega)$.

In conclusion,

the space $P^{\perp}$ in (2.54), as a closed subspace of $V^{\prime}$, can be isometrically identified with the space $\mathscr{H}$, as a closed subspace of $L_{2}(\Omega)$.

Thus, we return to (2.53) and (2.57); invoking further (2.58) and (2.63), we conclude that $\tilde{L}_{2}(\Omega)$ can be isometrically identified with $L_{2}(\Omega) / \mathscr{H}$ and (2.63) proves (2.49). 


\section{The space $\tilde{H}_{-1}(\Omega) \equiv\left[H^{1}(\Omega) \cap \mathscr{H}^{\perp}\right]^{\prime}$ and its properties}

The considerations of this section are critical in establishing the regularity of the second time derivative $w_{t t}$ of the Kirchhoff elastic or thermoelastic problems with clamped mechanical boundary conditions: see (1.5), (1.7), and (1.9), respectively; to be proved in Theorems 4.11 and 5.1, respectively.

3.1. The operator $A^{3 / 4} A_{\gamma}^{-1}$. With reference to the operator $A$ in (2.1), we recall that the space $\mathscr{D}\left(A^{3 / 4}\right)$ is given by (see [6])

$$
\mathscr{D}\left(A^{3 / 4}\right)=\left\{f \in H^{3}(\Omega):\left.f\right|_{\Gamma}=0,\left.\frac{\partial f}{\partial v}\right|_{\Gamma}=0\right\} \equiv H^{3}(\Omega) \cap H_{0}^{2}(\Omega),
$$

with equivalent norms, which complements the identifications in (2.3).

The counterpart of Lemma 2.2 is given next.

LEMmA 3.1. With reference to (2.1), (2.2), (2.6), and (3.1),

$$
\begin{gathered}
\mathscr{A}_{\gamma}: \text { continuous } \mathscr{D}\left(A^{3 / 4}\right) \equiv H^{3}(\Omega) \cap H_{0}^{2}(\Omega) \longrightarrow\left[H^{1}(\Omega) \cap \mathscr{H}^{\perp}\right] \\
\text { equivalently, } \mathscr{A}_{\gamma} A^{-3 / 4}: \text { continuous } L_{2}(\Omega) \longrightarrow\left[H^{1}(\Omega) \cap \mathscr{H}^{\perp}\right] .
\end{gathered}
$$

(a2) $\mathscr{A}_{\gamma} A^{-3 / 4}$ is injective (one-to-one) on $L_{2}(\Omega)$ :

$$
\mathscr{A}_{\gamma} A^{-3 / 4} x=0, \quad x \in L_{2}(\Omega) \Longrightarrow x=0
$$

(a3) For $F \in L_{2}(\Omega)$,

$$
\mathscr{A}_{\gamma}^{-1} F \in \mathscr{D}\left(A^{3 / 4}\right) \Longleftrightarrow A^{3 / 4} \mathscr{A}_{\gamma}^{-1} F \in L_{2}(\Omega) \Longleftrightarrow F \in\left[H^{1}(\Omega) \cap \mathscr{H}^{\perp}\right] .
$$

Thus, by the closed graph theorem, the operator $A^{3 / 4} \mathscr{A}_{\gamma}^{-1}$, as an operator on $L_{2}(\Omega)$, has the following domain:

$$
\mathscr{D}\left(A^{3 / 4} \mathscr{A}_{\gamma}^{-1}\right) \equiv\left[H^{1}(\Omega) \cap \mathscr{H}^{\perp}\right] .
$$

(a4) (Improving upon (a1) and (a3))

$\mathscr{A}_{\gamma}$ is an isomorphism from $\mathscr{D}\left(A^{3 / 4}\right) \equiv H^{3}(\Omega) \cap H_{0}^{2}(\Omega)$ onto $\left[H^{1}(\Omega) \cap \mathscr{H}^{\perp}\right]$,

equivalently, $\mathscr{A}_{\gamma} A^{-3 / 4}$ is an isomorphism from $L_{2}(\Omega)$ onto $\left[H^{1}(\Omega) \cap \mathscr{H}^{\perp}\right]$,

with bounded inverse

$$
\left(\mathscr{A}_{\gamma} A^{-3 / 4}\right)^{-1}=A^{3 / 4} \mathscr{A}_{\gamma}^{-1} \text { continuous from }\left[H^{1}(\Omega) \cap \mathscr{H}^{\perp}\right] \text { onto } L_{2}(\Omega) .
$$


By duality, we have, equivalently

$$
A^{-3 / 4} \mathscr{A}_{\gamma} \text { is an isomorphism from }\left[H^{1}(\Omega) \cap \mathscr{H}^{\perp}\right]^{\prime} \text { onto } L_{2}(\Omega),
$$

with bounded inverse

$$
\left(A^{-3 / 4} \mathscr{A}_{\gamma}\right)^{-1}=\mathscr{A}_{\gamma}^{-1} A^{3 / 4} \text { continuous from } L_{2}(\Omega) \text { onto }\left[H^{1}(\Omega) \cap \mathscr{H}^{\perp}\right]^{\prime} \text {. }
$$

Here [ ]' denotes the dual with respect to $L_{2}(\Omega)$.

(a5) The elliptic problem

$$
\begin{array}{r}
(1-\gamma \Delta) \psi=F \quad \text { in } \Omega ; \quad \text { or } \quad \mathscr{A}_{\gamma} \psi=F ; \\
\left.\psi\right|_{\Gamma}=0 \quad \text { on } \Gamma ;
\end{array}
$$

has a unique solution $\psi \in H^{3}(\Omega) \cap H_{0}^{2}(\Omega) \Longleftrightarrow F \in\left[H^{1}(\Omega) \cap \mathscr{H}^{\perp}\right]$.

Proof. (a1) Let $f \in H^{3}(\Omega) \cap H_{0}^{2}(\Omega) \equiv \mathscr{D}\left(A^{3 / 4}\right) \subset \mathscr{D}\left(\mathscr{A}_{\gamma}\right) \equiv H^{2}(\Omega) \cap H_{0}^{1}(\Omega)$, so that $F \equiv \mathscr{A}_{\gamma} f=(1-\gamma \Delta) f \in H^{1}(\Omega)$, as desired. Moreover, if $h \in \mathcal{H}$, see (2.5), since $f \in H_{0}^{2}(\Omega)$ in particular, then Green's identity (2.10) yields

$$
\begin{aligned}
(F, h)_{L_{2}(\Omega)} & =((1-\gamma \Delta) f, h)_{L_{2}(\Omega)} \\
& =(f,(1-\gamma \Delta) h)_{L_{2}(\Omega)}=0, \quad \forall h \in \mathscr{H},
\end{aligned}
$$

and then $F \in \mathscr{H}^{\perp}$ as well. Thus, $F \in\left[H^{1}(\Omega) \cap \mathscr{H}^{\perp}\right]$.

(a2) This is immediate, since $A^{-3 / 4} x \in \mathscr{D}\left(\mathscr{A}_{\gamma}\right)$ for $x \in L_{2}(\Omega)$, as noted above in (a1).

(a3) We first show that the right-hand side of (3.4) implies the left-hand side. Take at first $F \in H^{1}(\Omega)$ so that $\mathscr{A}_{\gamma}^{-1} F \in \mathscr{D}\left(\mathscr{A}_{\gamma}\right)$ (conservatively), and

$$
\psi \equiv \mathscr{A}_{\gamma}^{-1} F \Longleftrightarrow \mathscr{A}_{\gamma} \psi=F \quad \text { or } \quad \begin{cases}(1-\gamma \Delta) \psi \equiv F & \text { in } \Omega ; \\ \left.\psi\right|_{\Gamma}=0 & \text { on } \Gamma .\end{cases}
$$

Then elliptic theory [19] yields that $\psi \in H^{3}(\Omega) \cap H_{0}^{1}(\Omega)$. Next, we recall Lemma 2.2(a3) stating that for $F \in L_{2}(\Omega)$, then

$$
\left.\frac{\partial \psi}{\partial v}\right|_{\Gamma}=\left.\frac{\partial \mathscr{A}_{\gamma}^{-1} F}{\partial v}\right|_{\Gamma}=0 \Longleftrightarrow F \in \mathscr{H}^{\perp} .
$$

Thus, using $\Leftarrow$ in (3.14), we see that $F \in\left[H^{1}(\Omega) \cap \mathscr{H}^{\perp}\right]$ implies by the argument above that the solution of (3.13) satisfies $\psi \in H^{3}(\Omega) \cap H_{0}^{2}(\Omega)$. Thus, $\psi \equiv$ $\mathscr{A}_{\gamma}^{-1} F \in \mathscr{D}\left(A^{3 / 4}\right)$ by (3.1), and then $A^{3 / 4} \mathscr{A}_{\gamma}^{-1} F \in L_{2}(\Omega)$, as desired.

Conversely, we prove that the left-hand side of (3.4) implies the right-hand side. Let $A^{3 / 4} \psi \in L_{2}(\Omega)$ for $\psi \equiv \mathscr{A}_{\gamma}^{-1} F, F \in L_{2}(\Omega)$. Then, $\psi \in H^{3}(\Omega) \cap$ $H_{0}^{2}(\Omega)$ by (3.1). Next, the elliptic problem in (3.13) yields [19, page 188] that 
$F \in H^{1}(\Omega)$. Moreover, (3.14) this time from left to right $\Rightarrow$ yields that $F \in \mathscr{H}^{\perp}$. Hence, $F \in\left[H^{1}(\Omega) \cap \mathscr{H}^{\perp}\right]$, as desired.

(a4) Parts (a1) and (a3) yield part (a4).

(a5) Statement (3.11) is a PDE reformulation of (3.4).

Remark 3.2. The above argument in (a3) shows that for $F \in L_{2}(\Omega)$, then

$$
\begin{aligned}
& F \in\left[H_{0}^{1}(\Omega) \cap \mathscr{H}^{\perp}\right] \Longleftrightarrow \psi=\mathscr{A}_{\gamma}^{-1} F \in \mathscr{D}\left(\mathscr{A}_{\gamma}^{3 / 2}\right) \\
& \mathscr{D}\left(\mathscr{A}_{\gamma}^{3 / 2}\right)=\left\{f \in H^{3}(\Omega):\left.f\right|_{\Gamma}=0,\left.\Delta f\right|_{\Gamma}=0\right\} .
\end{aligned}
$$

3.2. The dual space $\left[H^{1}(\Omega) \cap \mathscr{H}^{\perp}\right]^{\prime}$ is isometric to the factor space $\left[H^{1}(\Omega)\right]^{\prime} / \mathbb{H}$. We first recall the space $\mathscr{H}$ of "generalized harmonic functions" defined in (2.5)

$$
\begin{aligned}
& \mathscr{H}=\mathcal{N}\{(1-\gamma \Delta)\}=\left\{h \in L_{2}(\Omega):(1-\gamma \Delta) h=0 \text { in } H^{-2}(\Omega)\right\}, \\
& L_{2}(\Omega)=\mathscr{H} \oplus \mathscr{H}^{\perp}, \text { where }(1-\gamma \Delta) \text { is viewed as an operator: } \\
& L_{2}(\Omega) \longrightarrow H^{-2}(\Omega) \equiv\left[\mathscr{D}\left(A^{1 / 2}\right)\right]^{\prime} .
\end{aligned}
$$

Next, we introduce a new closed space of "generalized harmonic functions" defined as

$$
\left\{\begin{array}{l}
\mathbb{H}=\mathcal{N}\{(1-\gamma \Delta)\}=\left\{h \in\left[H^{1}(\Omega)\right]^{\prime}:(1-\gamma \Delta) h=0 \text { in }\left[\mathscr{D}\left(A^{3 / 4}\right)\right]^{\prime}\right\} \\
=\left\{h \in\left[H^{1}(\Omega)\right]^{\prime}:((1-\gamma \Delta) h, \phi)_{L_{2}(\Omega)}=0\right. \\
\left.\quad \forall \phi \in H^{3}(\Omega) \cap H_{0}^{2}(\Omega) \equiv \mathscr{D}\left(A^{3 / 4}\right)\right\}, \\
\text { where here }(1-\gamma \Delta) \text { is viewed as an operator: }\left[H^{1}(\Omega)\right]^{\prime} \rightarrow\left[\mathscr{D}\left(A^{3 / 4}\right)\right]^{\prime}= \\
{\left[H^{3}(\Omega) \cap H_{0}^{2}(\Omega)\right]^{\prime} . \text { Moreover, }\left[H^{1}(\Omega)\right]^{\prime}=\mathbb{H} \oplus \mathbb{H}^{\perp} ; \pi\left[H^{1}(\Omega)\right]^{\prime}=\mathbb{H}^{\perp},} \\
\text { where } \pi=\pi^{*} \text { is the orthogonal projection of }\left[H^{1}(\Omega)\right]^{\prime} \text { onto } \mathbb{H}^{\perp} .
\end{array}\right.
$$

We note that $\mathscr{H} \subset \mathbb{H}$. In (3.16) and (3.17), $\mathcal{N}$ denotes "null space," while [ ]' is always duality with respect to $L_{2}(\Omega)$ as a pivot space. $\mathscr{H}$ is a closed subspace of $L_{2}(\Omega)$, while $\mathbb{H}$ is a closed subspace of $\left[H^{1}(\Omega)\right]^{\prime}$. Next we observe that

$$
\begin{aligned}
P & \equiv\left[H^{1}(\Omega) \cap \mathscr{H}^{\perp}\right] \\
& \equiv\left\{f \in H^{1}(\Omega): f \in \mathscr{H}^{\perp}\right\} \text { is a closed subspace of } H^{1}(\Omega) \equiv V .
\end{aligned}
$$

Indeed, let $f_{n} \in P$ so that $f_{n} \in H^{1}(\Omega)$ and $\left(f_{n}, h\right)_{L_{2}(\Omega)}=0$, for all $h \in \mathscr{H}$, and let $f_{n} \rightarrow f$ in $H^{1}(\Omega)$. Then $(f, h)_{L_{2}(\Omega)}=0$, for all $h \in \mathcal{H}$ and $f \in P$ as well. We next provide an isometric characterization of the dual space $P^{\prime}$, which is the counterpart of Proposition 2.5. 
Proposition 3.3. The space $\left[H^{1}(\Omega) \cap \mathscr{H}^{\perp}\right]^{\prime}$ dual of the space in (3.18) with respect to $L_{2}(\Omega)$ as a pivot space, is isometrically isomorphic (congruent, in the terminology of $\left[20\right.$, page 53]) to the factor (or quotient) space $\left[H^{1}(\Omega)\right]^{\prime} / \mathbb{H}$, where $\mathbb{H}$ is defined by (3.17). In symbols

$$
\left[H^{1}(\Omega) \cap \mathscr{H}^{\perp}\right]^{\prime} \cong\left[H^{1}(\Omega)\right]^{\prime} / \mathbb{H} \cong \mathbb{H}^{\perp} .
$$

For $g \in\left[H^{1}(\Omega) \cap \mathscr{H}^{\perp}\right]^{\prime}$,

$$
\|g\|_{\left[H^{1}(\Omega) \cap \mathscr{H}^{\perp}\right]^{\prime}}=\|[J g]\|_{\left[H^{1}(\Omega)\right]^{\prime} / \mathbb{H}}=\inf _{h \in \mathbb{H}}\|J g-h\|_{\left[H^{1}(\Omega)\right]^{\prime}}=\left\|g_{1}\right\|_{\left[H^{1}(\Omega)\right]^{\prime}},
$$

where $J$ denotes the isometric isomorphism from $\left[H^{1}(\Omega) \cap \mathscr{H}^{\perp}\right]^{\prime}$ onto $\left[H^{1}(\Omega)\right]^{\prime} / \mathbb{H}$ for the unique element $g_{1}=\pi g \in \mathbb{H}^{\perp}, g_{1} \in[\mathrm{Jg}]$ (the latter being the coset or equivalence class of $\left[H^{1}(\Omega)\right]^{\prime} / \mathbb{H}$ containing the element $J g$ ).

\section{Proof}

Step 1. Let $P$ be defined by (3.18), and $V \equiv H^{1}(\Omega)$ be defined by (3.18). By the standard result [1, Theorem 1.6, page 53], [20, Theorem 3.3, page 135], and [5, Theorem 6.11, page 118], we then have

$P^{\prime}$ is isometrically isomorphic (congruent) to the factor space $V^{\prime} / P^{\perp}$,

where

$$
\begin{aligned}
P^{\perp} & \equiv\left\{f \in V^{\prime}: f(v)=(f, v)_{V^{\prime} \times V}=0, \forall v \in P \subset V\right\} ; \\
P^{\prime} & \equiv \text { space of continuous linear functionals on } P ; \\
V^{\prime} & \equiv \text { space of continuous linear functionals on } V,
\end{aligned}
$$

and $(,)_{V^{\prime} \times V}$ denotes the duality pairing on $V^{\prime} \times V$.

We now take $L_{2}(\Omega)$ as a common pivot space. Accordingly, we have the identification

$$
V^{\prime}=\left[H^{1}(\Omega)\right]^{\prime}=\text { duality of } H^{1}(\Omega) \text { with respect to } L_{2}(\Omega) \text { as a pivot space. }
$$

We next find the corresponding isometric identification for $P^{\perp}$ (which is a closed subspace of $V^{\prime}$ ). We note the usual imbedding $V \subset L_{2}(\Omega) \subset V^{\prime}$, and we may identify the duality pairing $(,)_{V^{\prime} \times V}$ with the unique extension of the inner product of $L_{2}(\Omega)$ [1, Theorem 1.5, page 51]. Thus, the space $P^{\perp}$ in $V^{\prime}$ defined by (3.22) can be identified with the subspace of $\left[H^{1}(\Omega)\right]^{\prime}$ defined by

$$
P^{\perp}=\left\{f \in\left[H^{1}(\Omega)\right]^{\prime}:(f, v)_{L_{2}(\Omega)}=0, \forall v \in P \equiv\left[H^{1}(\Omega) \cap \mathscr{H}^{1}\right]\right\},
$$

and denoted by the same symbol. Since, in (3.26), we have, in particular, that $(h, v)_{L_{2}(\Omega)}=0$, for all $h \in \mathscr{H} \subset L_{2}(\Omega)$, we see at once that $\mathscr{H} \subset P^{\perp}$. 
462 Factor spaces and Kirchhoff equations

Step 2. With reference to $P^{\perp}$ in (3.26) and $\mathbb{H}$ in (3.17), we will now establish that

$$
P^{\perp}=\mathbb{H} \text {. }
$$

The proof will be based on Lemma 3.1(a5), that

$\psi$ runs over all of $H^{3}(\Omega) \cap H_{0}^{2}(\Omega)$ as $F$ runs over all of $\left[H^{1}(\Omega) \cap \mathscr{H}^{\perp}\right]$,

where $\psi$ solves the elliptic problem in (3.8) with right-hand side $F$.

Next, for any $h \in\left[H^{1}(\Omega)\right]^{\prime}$ and any $\psi \in H^{3}(\Omega) \cap H_{0}^{2}(\Omega)$, we can write

$$
((1-\gamma \Delta) h, \psi)_{L_{2}(\Omega)}=(h,(1-\gamma \Delta) \psi)_{L_{2}(\Omega)}
$$

by Green's identity (2.10). We now prove that

$$
\mathbb{H} \subset P^{\perp} .
$$

In fact, if $h \in \mathbb{H}$, then, in particular, $h \in\left[H^{1}(\Omega)\right]^{\prime}$ and by definition (3.17), we have that the left-hand side of (3.29) vanishes. Then, the right-hand side of (3.29) vanishes and hence we have that $(h, F)_{L_{2}(\Omega)}=0$ for $(1-\gamma \Delta) \psi=F$, where $F \in P \equiv\left[H^{1}(\Omega) \cap \mathscr{H}^{\perp}\right]$, see (3.18). Invoking (3.28), ultimately Lemma 3.1(a5), we then see that $h \in P^{\perp}$ by (3.26). Thus, (3.30) is established.

Conversely, we now show that

$$
P^{\perp} \subset \mathbb{H} \text {. }
$$

Indeed, let $h \in P^{\perp}$, so that $(h, F)_{L_{2}(\Omega)}=0$, for all $F \in P \equiv\left[H^{1}(\Omega) \cap \mathscr{H}^{\perp}\right]$ by (3.26). Then, $(h,(1-\gamma \Delta) \psi)_{L_{2}(\Omega)}=0$ for all $\psi \in H^{3}(\Omega) \cap H_{0}^{2}(\Omega)$ by (3.28). As a consequence of this, the left-hand side of (3.29) vanishes: ((1$\gamma \Delta) h, \psi)_{L_{2}(\Omega)}=0$, for all $\psi \in H^{3}(\Omega) \cap H_{0}^{2}(\Omega)$. Then $h \in \mathbb{H}$ by definition (3.17). Thus, (3.31) is established.

In conclusion, identity (3.27) is thus proved. Returning now to (3.21), with $P^{\perp}$ as in (3.27) and $V^{\prime}$ as in (3.25), we conclude that (3.19) holds true. Proposition 3.3 is established.

Remark 3.4. Complementing (3.8), we have

$$
A^{-3 / 4} \mathscr{A}_{\gamma} h=0, \quad \text { iff } h \in \mathbb{H} \text {. }
$$

Indeed, let $f \in L_{2}(\Omega)$, equivalently $\phi=A^{-3 / 4} f \in H^{3}(\Omega) \cap H_{0}^{2}(\Omega)$. Then, the above statement holds true if and only if

$$
\begin{aligned}
0 & =\left(A^{-3 / 4} \mathscr{A}_{\gamma} h, f\right)_{L_{2}(\Omega)}=\left(h, \mathscr{A}_{\gamma} \phi\right)_{L_{2}(\Omega)}=(h,(1-\gamma \Delta) \phi)_{L_{2}(\Omega)} \\
& =((1-\gamma \Delta) h, \phi)_{L_{2}(\Omega)}, \quad \forall \phi \in H^{3}(\Omega) \cap H_{0}^{2}(\Omega),
\end{aligned}
$$

by use of (2.10), hence $(1-\gamma \Delta) h=0$ and $h \in \mathbb{H}$, by definition (3.17). 
3.3. Definition of the space $\tilde{H}_{-1}(\Omega)$. Equivalent formulations: $\tilde{H}_{-1}(\Omega) \equiv$ $\left[H^{1}(\Omega) \cap H^{\perp}\right]^{\prime}$. Paralleling the development of Section 2.2, we consider (see (2.1), (2.2), (2.3), (2.4), and (3.1))

(i)

$$
\begin{aligned}
& \text { the space } \mathscr{D}\left(A^{3 / 4}\right) \equiv H^{3}(\Omega) \cap H_{0}^{2}(\Omega) \\
& \text { as a closed subspace of } \mathscr{D}\left(\mathscr{A}_{\gamma}\right)=H^{2}(\Omega) \cap H_{0}^{1}(\Omega) \text {; }
\end{aligned}
$$

(ii) the space $\mathscr{D}\left(\mathscr{A}_{\gamma}^{1 / 2}\right)$ as a pivot space, with norm as in (2.4), or (2.28).

The space $\mathscr{D}\left(A^{3 / 4}\right)$ is dense in $\mathscr{D}\left(\mathscr{A}_{\gamma}^{1 / 2}\right)$, however, so [1, page 51] applies. We then define the (Hilbert) space $\tilde{H}_{-1}(\Omega)$ as follows:

$$
\begin{aligned}
\tilde{H}_{-1}(\Omega) \equiv & \text { dual of the space } \mathscr{D}\left(A^{3 / 4}\right) \text { with respect to the space } \mathscr{D}\left(\mathscr{A}_{\gamma}^{1 / 2}\right) \\
& \text { as a pivot space, endowed with the norm of (2.28). }
\end{aligned}
$$

This means the following: let $f \in \mathscr{D}\left(A^{3 / 4}\right) \equiv H^{3}(\Omega) \cap H_{0}^{2}(\Omega) \subset \mathscr{D}\left(\mathscr{A}_{\gamma}\right) \equiv$ $H^{2}(\Omega) \cap H_{0}^{1}(\Omega)$, or $\phi=A^{3 / 4} f \in L_{2}(\Omega)$. Then

$$
\begin{aligned}
g \in \tilde{H}_{-1}(\Omega) \Longleftrightarrow(f, g)_{\mathscr{D}\left(\mathscr{A}_{\gamma}^{1 / 2}\right)} & \left(\mathscr{A}_{\gamma} f, g\right)_{L_{2}(\Omega)}=\text { finite } \\
& \forall f \in \mathscr{D}\left(A^{3 / 4}\right)=H^{3}(\Omega) \cap H_{0}^{2}(\Omega), \\
= & \left(f, \mathscr{A}_{\gamma} g\right)_{L_{2}(\Omega)}=\left(A^{-3 / 4} \phi, \mathscr{A}_{\gamma} g\right)_{L_{2}(\Omega)} \\
= & \left(\phi, A^{-3 / 4} \mathscr{A}_{\gamma} g\right)_{L_{2}(\Omega)}=\text { finite } \quad \forall \phi \in L_{2}(\Omega),
\end{aligned}
$$

where we write the same way inner products and corresponding duality pairings.

Proposition 3.5. (i) Definition (3.36) is equivalent to the following restatement:

$$
\begin{aligned}
g \in \tilde{H}_{-1}(\Omega) \Longleftrightarrow\left(\mathscr{A}_{\gamma} f, g\right)_{L_{2}(\Omega)} & =(F, g)_{L_{2}(\Omega)} \\
& =((1-\gamma \Delta) f, g)_{L_{2}(\Omega)} \\
& =(f,(1-\gamma \Delta) g)_{L_{2}(\Omega)}=\text { finite }
\end{aligned}
$$

for all $f \in \mathscr{D}\left(A^{3 / 4}\right) \equiv H^{3}(\Omega) \cap H_{0}^{2}(\Omega)$, or for all $F \in H^{1}(\Omega) \cap \mathscr{H}$, where $F=\mathscr{A}_{\gamma} f=(1-\gamma \Delta) f$.

(ii) Accordingly,

$$
\tilde{H}_{-1}(\Omega) \equiv\left[H^{1}(\Omega) \cap \mathscr{H}^{\perp}\right]^{\prime} \cong\left[H^{1}(\Omega)\right]^{\prime} / \mathbb{H} \cong \mathbb{H}^{\perp},
$$

with duality with respect to $L_{2}(\Omega)$ as a pivot space. 
464 Factor spaces and Kirchhoff equations

(iii) Definition (3.36) is equivalent to the following restatement:

$$
g \in \tilde{H}_{-1}(\Omega) \Longleftrightarrow A^{-3 / 4} \mathscr{A}_{\gamma} g \in L_{2}(\Omega) .
$$

(Recall also Remark 3.4.)

(iv) (Counterpart of Proposition 2.5)

$$
g \in \tilde{H}_{-1}(\Omega) \Longleftrightarrow\left\{\begin{array}{l}
g \text { has a component } g_{1} \text { defined by } \\
g_{1}=\pi g=\left.g\right|_{\mathbb{H}^{\perp}} \in \mathbb{H}^{\perp} \subset\left[H^{1}(\Omega)\right]^{\prime} \\
\text { which is the orthogonal projection of } g \text { onto } \mathbb{H}^{\perp},
\end{array}\right.
$$

in which case

$$
(1-\gamma \Delta) g=(1-\gamma \Delta) g_{1} \quad \text { in }\left[\mathscr{D}\left(A^{3 / 4}\right)\right]^{\prime}
$$

Proof. (i) Returning to (3.36), we invoke Lemma 3.1(a4), (3.6a) and obtain that $\mathscr{A}_{\gamma} \mathscr{D}\left(A^{3 / 4}\right)=\left[H^{1}(\Omega) \cap \mathscr{H}^{\perp}\right]$. Thus, (3.36) yields (3.37) also via (2.10).

(ii) Part (i) yields the first identity in (3.38), and then (3.19) of Proposition 3.3 completes the proof of (3.38).

(iii) Part (iii), (3.39), follows at once from (3.36b).

(iv) Counterpart of the proof of Proposition 2.5. Since $g \in \tilde{H}_{-1}(\Omega)$ implies a fortiori $g \in\left[H^{1}(\Omega)\right]^{\prime}$ by (3.38), then (3.17) implies (3.40) so that

$$
\begin{aligned}
((1-\gamma \Delta) f, g)_{L_{2}(\Omega)} & =\left(\mathscr{A}_{\gamma} f, g\right)_{L_{2}(\Omega)}=\left(\mathscr{A}_{\gamma} f, g_{1}\right)_{L_{2}(\Omega)} \\
& =\left((1-\gamma \Delta) f, g_{1}\right)_{L_{2}(\Omega)},
\end{aligned}
$$

for all $f \in H^{3}(\Omega) \cap H_{0}^{2}(\Omega)$ so that $\mathscr{A}_{\gamma} f \in\left[H^{1}(\Omega) \cap \mathscr{H}^{\perp}\right]$ by (3.6a). Hence, (3.42) and (2.10) yield

$$
(f,(1-\gamma \Delta) g)_{L_{2}(\Omega)}=\left(f,(1-\gamma \Delta) g_{1}\right)_{L_{2}(\Omega)},
$$

and (3.41) is established.

\section{Implications on regularity of Kirchhoff elastic plate equations with clamped BC}

4.1. PDE model. In this section, we examine the implications of the main results of Sections 2 and 3 on the sharp regularity of solutions to the following Kirchhoff elastic mixed problem with clamped BC:

$$
\begin{array}{ll}
w_{t t}-\gamma \Delta w_{t t}+\Delta^{2} w=F & \text { in }(0, T] \times \Omega \equiv Q ; \\
w(0, \cdot)=w_{0}, \quad w_{t}(0, \cdot)=w_{1} & \text { in } \Omega \\
\left.w\right|_{\Sigma} \equiv 0,\left.\quad \frac{\partial w}{\partial v}\right|_{\Sigma} \equiv u & \text { in }(0, T] \times \Gamma \equiv \Sigma .
\end{array}
$$


In (4.1), $\Omega$ is an open bounded domain in $\mathbb{R}^{n}$, with smooth boundary $\Gamma$, and $F$ and $u$ are forcing terms to be specified below. Moreover, throughout this section, $\gamma$ is a positive constant, $\gamma>0$.

Abstract model. The abstract model of the mixed problem (4.1) is given by (see $[12,16,21,22])$,

$$
(I+\gamma \mathscr{A}) w_{t t}=-A w+A G_{2} u+F
$$

where $A$ and $\mathscr{A}$ are the operators defined in (2.1) and (2.2), respectively. Moreover, $G_{2}$ in (4.2) is the following Green's map defined by [12],

$$
v=G_{2} u \Longleftrightarrow\left\{\Delta^{2} v=0 \text { in } \Omega ;\left.v\right|_{\Gamma}=0,\left.\frac{\partial v}{\partial v}\right|_{\Gamma}=u\right\},
$$

and by elliptic regularity [6, 19], see also [12, 22],

$$
\begin{gathered}
G_{2} \text { : continuous } L_{2}(\Gamma) \\
\longrightarrow H^{3 / 2}(\Omega) \cap H_{0}^{1}(\Omega) \subset H^{3 / 2-4 \epsilon}(\Omega) \cap H_{0}^{1}(\Omega)=\mathscr{D}\left(A^{3 / 8-\epsilon}\right) \\
A^{3 / 8-\epsilon} G_{2} \text { : continuous } L_{2}(\Gamma) \longrightarrow L_{2}(\Omega) .
\end{gathered}
$$

Next, we will consider separately the boundary homogeneous $(u \equiv 0)$ and nonhomogeneous case $(u \not \equiv 0)$.

4.2. The boundary homogeneous case: $u \equiv 0$. When $u \equiv 0$ in (4.1b), we may rewrite the abstract model (4.2) as the following first-order abstract equation

$$
\frac{d}{d t}\left[\begin{array}{c}
w \\
w_{t}
\end{array}\right]=\mathbb{A}_{0, \gamma}\left[\begin{array}{c}
w \\
w_{t}
\end{array}\right]+\left[\begin{array}{c}
0 \\
\mathscr{A}_{\gamma}^{-1} F
\end{array}\right],
$$

where the operator $Y_{\gamma} \supset \mathscr{D}\left(\mathbb{A}_{0, \gamma}\right) \rightarrow Y_{\gamma}$ is given by

$$
\begin{gathered}
\mathbb{A}_{0, \gamma}=\left[\begin{array}{cc}
0 & I \\
-\mathscr{A}_{\gamma}^{-1} A & 0
\end{array}\right] ; \quad \mathbb{A}_{0, \gamma}^{*}=\left[\begin{array}{cc}
0 & -I \\
\mathscr{A}_{\gamma}^{-1} A & 0
\end{array}\right] ; \\
\mathscr{D}\left(\mathbb{A}_{0, \gamma}\right) \equiv \mathscr{D}\left(\mathbb{A}_{0, \gamma}^{*}\right) \equiv \mathscr{D}\left(A^{3 / 4}\right) \times \mathscr{D}\left(A^{1 / 2}\right) \\
\equiv\left[H^{3}(\Omega) \cap H_{0}^{2}(\Omega)\right] \times H_{0}^{2}(\Omega) ; \\
Y_{\gamma} \equiv \mathscr{D}\left(A^{1 / 2}\right) \times \mathscr{D}\left(\mathscr{A}_{\gamma}^{1 / 2}\right) \equiv H_{0}^{2}(\Omega) \times H_{0}^{1}(\Omega)
\end{gathered}
$$

(norm equivalence). The $*$ denotes the $Y_{\gamma}$-adjoint. In identifying the domains in (4.7), we have recalled from (2.3) that $\mathcal{A}_{\gamma}^{-1 / 2} A^{1 / 4}$ is an isomorphism (bounded, with bounded inverse) on $L_{2}(\Omega)$. The following well-known and elementary result holds true. 
Lemma 4.1. The operator $-\mathscr{A}_{\gamma}^{-1} A$ is negative self-adjoint on the space $\mathscr{D}\left(\mathscr{A}_{\gamma}^{1 / 2}\right)$ topologized as in (2.4). Consequently, the operator $\mathbb{A}_{0, \gamma}$ in (4.6) is skew-adjoint: $\mathbb{A}_{0, \gamma}^{*}=-\mathbb{A}_{0, \gamma}$ and is the generator of a s.c. unitary group $e^{\mathbb{A}_{0, \gamma} t}$ on the space $Y_{\gamma}$ defined in (4.8).

Thus, the solution of problem (4.5) — that is of problem (4.1) with $u \equiv 0$-is given by

$$
\left[\begin{array}{c}
w(t) \\
w_{t}(t)
\end{array}\right]=e^{\mathbb{A}_{0, \gamma} t}\left[\begin{array}{c}
w_{0} \\
w_{1}
\end{array}\right]+\int_{0}^{t} e^{\mathbb{A}_{0, \gamma}(t-\tau)}\left[\begin{array}{c}
0 \\
\mathscr{A}_{\gamma}^{-1} F(\tau)
\end{array}\right] d \tau,
$$

under appropriate assumptions on the initial data $\left\{w_{0}, w_{1}\right\}$ and on the forcing term $F$ (see below), as to justify the validity of (4.9). To this end, the following lemma provides the first key step, and produces subspaces invariant for the free dynamics.

LEMMA 4.2. With $Y_{\gamma} \equiv \mathscr{D}\left(A^{1 / 2}\right) \times \mathscr{D}\left(\mathscr{A}_{\gamma}^{1 / 2}\right)$ as in (4.8), the following identities hold true:

$$
\begin{aligned}
& \mathscr{D}\left(\mathbb{A}_{0, \gamma}\right)=\mathscr{D}\left(\mathbb{A}_{0, \gamma}^{*}\right)=\mathscr{D}\left(A^{3 / 4}\right) \times \mathscr{D}\left(A^{1 / 2}\right) ; \\
& \mathscr{D}\left(\mathbb{A}_{0, \gamma}^{2}\right) \equiv \mathscr{D}\left(\left(\mathbb{A}_{0,2}^{*}\right)^{2}\right)=\mathscr{D}\left(A^{1 / 2} \mathscr{A}_{\gamma}^{-1} A\right) \times \mathscr{D}\left(A^{3 / 4}\right) ; \\
& \mathscr{D}\left(A^{1 / 2} \mathscr{A}_{\gamma}^{-1} A\right)=A^{-1} \mathscr{H}^{\perp} \cong A^{-1} \tilde{L}_{2}(\Omega), \quad \text { by }(2.49) .
\end{aligned}
$$

Consequently, if []$^{\prime}$ denotes duality with respect to $Y_{\gamma}$ as a pivot space,

$$
\begin{aligned}
& {\left[\mathscr{D}\left(\mathbb{A}_{0, \gamma}\right)\right]^{\prime} \equiv\left[\mathscr{D}\left(\mathbb{A}_{0, \gamma}^{*}\right)\right]^{\prime} \equiv \mathscr{D}\left(A^{1 / 4}\right) \times \tilde{L}_{2}(\Omega) ;} \\
& {\left[\mathscr{D}\left(\mathbb{A}_{0, \gamma}^{2}\right)\right]^{\prime} \equiv\left[\mathscr{D}\left(\left(\mathbb{A}_{0, \gamma}^{*}\right)^{2}\right)\right]^{\prime} \equiv \tilde{L}_{2}(\Omega) \times \tilde{H}_{-1}(\Omega),}
\end{aligned}
$$

where the spaces in (4.13) are defined in (2.29) and (3.35), respectively.

Proof. First, $\mathscr{D}\left(\mathbb{A}_{0, \gamma}\right)=\mathscr{D}\left(\mathbb{A}_{0, \gamma}^{*}\right)$ was identified in (4.7), then, duality gives (4.12) by invoking the definition (2.29) of $\tilde{L}_{2}(\Omega)$. Next, by (4.6),

$$
\mathbb{A}_{0, \gamma}^{2}\left[\begin{array}{l}
x_{1} \\
x_{2}
\end{array}\right]=\mathbb{A}_{0, \gamma}\left[\begin{array}{c}
x_{2} \\
-\mathscr{A}_{\gamma}^{-1} A x_{1}
\end{array}\right]=\left[\begin{array}{c}
-\mathscr{A}_{\gamma}^{-1} A x_{1} \\
-\mathscr{A}_{\gamma}^{-1} A x_{2}
\end{array}\right] \in Y_{\gamma}=\left[\begin{array}{c}
\mathscr{D}\left(A^{1 / 2}\right) \\
\times \\
\mathscr{D}\left(\mathscr{A}_{\gamma}^{1 / 2}\right)
\end{array}\right],
$$

if and only if

$$
\begin{gathered}
A^{1 / 2} \mathscr{A}_{\gamma}^{-1} A x_{1} \in L_{2}(\Omega) \Longleftrightarrow A x_{1} \in \mathscr{H}^{\perp}, \quad \text { by Lemma } 2.2(\mathrm{a} 3) ; \\
\mathscr{A}_{\gamma}^{-1 / 2} A x_{2} \in L_{2}(\Omega) \Longleftrightarrow x_{2} \in \mathscr{D}\left(A^{3 / 4}\right), \quad \text { by }(2.3),
\end{gathered}
$$


as $\mathscr{A}_{\gamma}^{-1 / 2} A^{1 / 4}$ is an isomorphism on $L_{2}(\Omega)$. Then (4.15) and (2.49) establish (4.10) and (4.11) for $\mathbb{A}_{0, \gamma}^{2}$. Similarly, for $\left(\mathbb{A}_{0, \gamma}^{*}\right)^{2}$.

Furthermore, it follows from definition (3.35) that the second component space of $\left[\mathscr{D}\left(\mathbb{A}_{0, \gamma}^{2}\right)\right]^{\prime}=\left[\mathscr{D}\left(\left(\mathbb{A}_{0, \gamma}^{*}\right)^{2}\right)\right]^{\prime}$ is precisely $\tilde{H}_{-1}(\Omega)$, as claimed in (4.12).

Finally, in order to identify the first component space of, say, $\left[\mathscr{D}\left(\mathbb{A}_{0, \gamma}^{2}\right)\right]^{\prime}$, we proceed as follows: let

$$
\begin{aligned}
& f \in A^{-1} \mathscr{H}^{\perp}, \text { equivalently } A f=\tilde{h} \in \mathscr{H}^{\perp} \\
& g \in \text { first component space of }\left[\mathscr{D}\left(\mathbb{A}_{0, \gamma}^{2}\right)\right]^{\prime} .
\end{aligned}
$$

Then, by definition of duality in the first component space $A^{-1} \mathscr{H} \perp$ of $\mathscr{D}\left(\mathbb{A}_{0, \gamma}^{2}\right)$ with respect to the first component space $\mathscr{D}\left(A^{1 / 2}\right)$ of $Y_{\gamma}$, we have via (4.16) that

$$
(f, g)_{\mathscr{D}\left(A^{1 / 2}\right)}=(A f, g)_{L_{2}(\Omega)}=(\tilde{h}, g)_{L_{2}(\Omega)}=\text { finite },
$$

for all $\tilde{h} \in \mathscr{H}^{\perp}$. Then, by (2.31), it follows that (4.17) holds true if and only if $g \in \tilde{L}_{2}(\Omega)$. Thus, $\tilde{L}_{2}(\Omega)$ is the first component space of $\left[\mathscr{D}\left(\mathbb{A}_{0, \gamma}^{2}\right)\right]^{\prime}$, as claimed in (4.13).

On the basis of Lemma 4.2, we may now proceed to justify the solution formula (4.9) and obtain well-posedness results for (4.1) with $u \equiv 0$, under various circumstances. We collect, for completeness, known and new results.

To begin with, the main novelty of the next result, Proposition 4.3 , is in asserting the regularity result for $w_{t t}$ in part (c), (4.24), which complements and refines [21, Proposition 3.4(b), equation (3.46c)]. The rest of Proposition 4.3 is more or less known [21, Proposition 3.4(b), page 415] and [7, page 123] for the trace in (4.21).

Proposition 4.3 (well-posedness of (4.5) on $Y_{\gamma}$ ). With reference to problem (4.1) with $u \equiv 0$, let (norm equivalence)

(a)

$$
\begin{gathered}
\left\{w_{0}, w_{1}\right\} \in Y_{\gamma} \equiv \mathscr{D}\left(A^{1 / 2}\right) \times \mathscr{D}\left(\mathscr{A}_{\gamma}^{1 / 2}\right) \equiv H_{0}^{2}(\Omega) \times H_{0}^{1}(\Omega) ; \\
F \in L_{1}\left(0, T ; H^{-1}(\Omega)\right), \quad H^{-1}(\Omega)=\left[\mathscr{D}\left(\mathscr{A}_{\gamma}^{1 / 2}\right)\right]^{\prime}=\left[\mathscr{D}\left(A^{1 / 4}\right)\right]^{\prime},
\end{gathered}
$$

duality with respect to $L_{2}(\Omega)$. Then, continuously

$$
\begin{gathered}
\left\{w, w_{t}\right\} \in C\left([0, T] ; Y_{\gamma} \equiv H_{0}^{2}(\Omega) \times H_{0}^{1}(\Omega) \equiv \mathscr{D}\left(A^{1 / 2}\right) \times \mathscr{D}\left(A^{1 / 4}\right)\right), \\
\left.\Delta w\right|_{\Sigma} \in L_{2}(\Sigma) \equiv L_{2}\left(0, T ; L_{2}(\Gamma)\right) ; \quad w_{t t} \in L_{1}\left(0, T ; \tilde{L}_{2}(\Omega)\right) .
\end{gathered}
$$

(b) Assume more specifically that $F \in L_{2}\left(0, T ; H^{-1}(\Omega)\right)$. Then continuously,

$$
w_{t t} \in L_{2}\left(0, T ; L_{2}(\Omega)\right) .
$$


(c) Next, assume $\left\{w_{0}, w_{1}\right\} \in Y_{\gamma}$ as in (4.18) and, moreover,

$$
F \in L_{1}\left(0, T ; H^{-1}(\Omega)\right) \cap C\left([0, T] ; H^{-2}(\Omega)\right), \quad H^{-2}(\Omega)=\left[\mathscr{D}\left(A^{1 / 2}\right)\right]^{\prime} .
$$

Then, continuously (see (2.29)),

$$
w_{t t} \in C\left([0, T] ; \tilde{L}_{2}(\Omega)\right)
$$

Proof. (a) By (4.19) we have $\mathscr{A}_{\gamma}^{-1} F \in L_{1}\left(0, T ; \mathscr{D}\left(\mathscr{A}_{\gamma}^{1 / 2}\right)\right)$, hence $\left[0, \mathscr{A}_{\gamma}^{-1} F\right] \in$ $L_{1}\left(0, T ; Y_{\gamma}\right)$ by (4.8). Then, part (a), (4.20), for $\left\{w, w_{t}\right\}$ follows at once from (4.9).

Moreover, the sharp trace regularity (4.21) for $\left.\Delta w\right|_{\Sigma}$ is shown in [7, equation (2.24), page 123], when $F \equiv 0$. The same proof, with suitable complements, continues to apply and shows (4.21) for $\left.\Delta w\right|_{\Sigma}$ when, in addition, $F \in L_{1}\left(0, T ; H^{-1}(\Omega)\right)$ (see [4, implication (3.97)-(3.99); and (C.48), (C.49) of Appendix C]).

Finally, (4.19) yields $A^{-1 / 2} F \in L_{1}\left(0, T ; \mathscr{D}\left(A^{1 / 4}\right)\right)$ and (4.20) yields $A^{1 / 2} w \in$ $C\left([0, T] ; L_{2}(\Omega)\right)$; thus, (4.2) with $u \equiv 0$ yields

$$
A^{-1 / 2} \mathscr{A}_{\gamma} w_{t t}=-A^{1 / 2} w+A^{-1 / 2} F \in L_{1}\left(0, T ; L_{2}(\Omega)\right)
$$

and then the characterization $(2.33)$ of $\tilde{L}_{2}(\Omega)$ yields

$$
w_{t t} \in L_{1}\left(0, T ; \tilde{L}_{2}(\Omega)\right)
$$

as claimed in (4.21).

(b) The regularity (4.22) for $w_{t t}$ was established in [18, page 43], at least when $F \equiv 0$, precisely as a consequence of (4.21) for $\left.\Delta w\right|_{\Sigma}$, and this argument continues to hold true for $F \in L_{2}\left(0, T ; H^{-1}(\Omega)\right)$. See also [4, equations (7.7) and (9.14b)] for $F \in L_{2}\left(0, T ; H^{-1}(\Omega)\right)$.

(c) Part (c) is a new result, which will be established as a consequence of Section 2. First, we have that $A^{1 / 2} w \in C\left([0, T] ; L_{2}(\Omega)\right)$ by part (a), (4.20), via the regularity of $F$ in (4.23) (left), and, moreover, $A^{-1 / 2} F \in C\left([0, T] ; L_{2}(\Omega)\right.$ ) by (4.23) (right). Next, returning to (4.2) with $u \equiv 0$, we can write by the above regularity $A^{-1 / 2} \mathscr{A}_{\gamma} w_{t t} \in C\left([0, T] ; L_{2}(\Omega)\right)$. Invoking the characterization (2.33) of $\tilde{L}_{2}(\Omega)$, we conclude that (4.25) holds true if and only if $w_{t t} \in$ $C\left([0, T] ; \tilde{L}_{2}(\Omega)\right)$ and (4.24) is established.

The next result is in [21, Proposition 3.4(a), page 415], save for the new information and clarification provided by Lemma 2.2(a3) on $A^{1 / 2} \mathscr{A}_{\gamma}^{-1}$. 
Proposition 4.4 (well-posedness of (4.5) on $\mathscr{D}\left(\mathbb{A}_{0, \gamma}\right)$ ). With reference to problem (4.1) with $u \equiv 0$, let (norm equivalence (2.3) and (3.1)),

$$
\begin{aligned}
\left\{w_{0}, w_{1}\right\} \in \mathscr{D}\left(\mathbb{A}_{0, \gamma}\right) & =\mathscr{D}\left(A^{3 / 4}\right) \times \mathscr{D}\left(A^{1 / 2}\right) \\
& =\left[H^{3}(\Omega) \cap H_{0}^{2}(\Omega)\right] \times H_{0}^{2}(\Omega) ;
\end{aligned}
$$

$$
F \in L_{1}\left(0, T ; \mathscr{H}^{\perp}\right) \text {, equivalently } \mathscr{A}_{\gamma}^{-1} F \in L_{1}\left(0, T ; \mathscr{D}\left(A^{1 / 2}\right)\right)
$$

(recall Lemma 2.2(a3)). Then, continuously,

$$
\begin{gathered}
\left\{w, w_{t}\right\} \in C\left([0, T] ; \mathscr{D}\left(\mathbb{A}_{0, \gamma}\right)=\mathscr{D}\left(A^{3 / 4}\right) \times \mathscr{D}\left(A^{1 / 2}\right)\right) \\
\equiv C\left([0, T] ;\left[H^{3}(\Omega) \cap H_{0}^{2}(\Omega)\right] \times H_{0}^{2}(\Omega)\right) ; \\
w_{t t}=-\mathscr{A}_{\gamma}^{-1} A w+\mathscr{A}_{\gamma}^{-1} F \in L_{1}\left(0, T ; H_{0}^{1}(\Omega)=\mathscr{D}\left(A^{1 / 4}\right)\right) ; \\
\mathscr{A}_{\gamma}^{-1} A w \in C\left([0, T] ; \mathscr{D}\left(\mathscr{A}_{\gamma}^{1 / 2}\right) \equiv H_{0}^{1}(\Omega)\right) ; \\
\mathscr{A}_{\gamma}^{-1} F \in L_{1}\left(0, T ; \mathscr{D}\left(A^{1 / 2}\right) \equiv H_{0}^{2}(\Omega)\right) .
\end{gathered}
$$

Proof. By (4.27) we have $\left[0, \mathscr{A}^{-1} F\right] \in L_{1}\left(0, T ; \mathscr{D}\left(\mathbb{A}_{0, \gamma}\right)\right)$ via (4.10). Then, (4.28) follows by (4.9) via (4.26) as well. Then (4.27), (4.28), and (4.2) with $u \equiv 0$ readily yield (4.29) and (4.30), since $\mathscr{A}_{\gamma}^{-1 / 2} A^{1 / 4}$ is an isomorphism on $L_{2}(\Omega)$, by $(2.3)$.

Proposition 4.5 (well-posedness of $(4.5)$ on $\left.\left[\mathscr{D}\left(A_{0, \gamma}^{*}\right)\right]^{\prime}\right)$. With reference to problem (4.1) with $u \equiv 0$, let (norm equivalence (2.3))

(a)

$$
\begin{gathered}
\left\{w_{0}, w_{1}\right\} \in\left[\mathscr{D}\left(A_{0, \gamma}^{*}\right)\right]^{\prime} \equiv \mathscr{D}\left(A^{1 / 4}\right) \times \tilde{L}_{2}(\Omega) \equiv H_{0}^{1}(\Omega) \times \tilde{L}_{2}(\Omega) ; \\
F \in L_{1}\left(0, T ; H^{-2}(\Omega)\right), \text { equivalently } A^{-1 / 2} F \in L_{1}\left(0, T ; L_{2}(\Omega)\right),
\end{gathered}
$$

recalling $\mathscr{D}\left(A^{1 / 2}\right) \equiv H_{0}^{2}(\Omega)$ from (2.3). Then, continuously,

$$
\left\{w, w_{t}\right\} \in C\left([0, T] ;\left[\mathscr{D}\left(\mathbb{A}_{0, \gamma}^{*}\right)\right]^{\prime} \equiv \mathscr{D}\left(A^{1 / 4}\right) \times \tilde{L}_{2}(\Omega) \equiv H_{0}^{1}(\Omega) \times \tilde{L}_{2}(\Omega)\right) .
$$

(b) Next, assume (4.26) for $\left\{w_{0}, w_{1}\right\}$ and moreover,

$$
\begin{aligned}
& F \in L_{1}\left(0, T ; H^{-2}(\Omega)\right) \cap C\left([0, T] ;\left[\mathscr{D}\left(A^{3 / 4}\right)\right]^{\prime}\right) \\
& \left(\text { respectively, } F \in L_{1}\left(0, T ; H^{-2}(\Omega)\right) \cap L_{2}\left(0, T ;\left[\mathscr{D}\left(A^{3 / 4}\right)\right]^{\prime}\right)\right),
\end{aligned}
$$

duality with respect to $L_{2}(\Omega)$. Then, continuously,

$$
w_{t t} \in C\left([0, T] ; \tilde{H}_{-1}(\Omega)\right),\left(\text { respectively, } w_{t t} \in L_{2}\left(0, T ; \tilde{H}_{-1}(\Omega)\right)\right),
$$

with $\tilde{H}_{-1}(\Omega)$ the space defined in (3.35). 
Proof. (a) By (2.33) we have that $\mathscr{A}_{\gamma}^{-1} F \in L_{1}\left(0, T ; \tilde{L}_{2}(\Omega)\right)$ if and only if $A^{-1 / 2} \mathscr{A}_{\gamma} A_{\gamma}^{-1} F=A^{-1 / 2} F \in L_{1}\left(0, T ; L_{2}(\Omega)\right)$, as guaranteed by assumption (4.32). Thus, by (4.12),

$$
\left[\begin{array}{c}
0 \\
\mathscr{A}_{\gamma}^{-1} F
\end{array}\right] \in L_{1}\left(0, T ;\left[\mathscr{D}\left(\mathbb{A}_{0, \gamma}^{*}\right)\right]^{\prime}\right),
$$

or equivalently, via (4.8),

$$
\begin{aligned}
\mathbb{A}_{0, \gamma}^{-1}\left[\begin{array}{c}
0 \\
\mathscr{A}_{\gamma}^{-1} F
\end{array}\right] & =\left[\begin{array}{cc}
0 & -A^{-1} \mathscr{A}_{\gamma} \\
I & 0
\end{array}\right]\left[\begin{array}{c}
0 \\
\mathscr{A}_{\gamma}^{-1} F
\end{array}\right] \\
& =\left[\begin{array}{c}
-A^{-1} F \\
0
\end{array}\right] \in L_{1}\left(0, T ; Y_{\gamma}\right) .
\end{aligned}
$$

Thus, in view of (4.36) and (4.31), we obtain via (4.9),

$$
\begin{aligned}
& \mathbb{A}_{0, \gamma}^{-1}\left[\begin{array}{c}
w(t) \\
w_{t}(t)
\end{array}\right]=e^{\mathbb{A}_{0, \gamma} t} \mathbb{A}_{0, \gamma}^{-1}\left[\begin{array}{l}
w_{0} \\
w_{1}
\end{array}\right] \\
& +\int_{0}^{t} e^{\mathbb{A}_{0, \gamma}(t-\tau)} \mathbb{A}_{0, \gamma}^{-1}\left[\begin{array}{c}
0 \\
\mathscr{A}_{\gamma}^{-1} F(\tau)
\end{array}\right] d \tau \in C\left([0, T] ; Y_{\gamma}\right),
\end{aligned}
$$

and (4.37) proves (4.33), via (4.12).

(b) First, we have that $A^{1 / 4} w \in C\left([0, T] ; L_{2}(\Omega)\right)$ by part (a), (4.33) via the regularity of $F$ in (4.34) (left), and, moreover, $A^{-3 / 4} F \in C\left([0, T] ; L_{2}(\Omega)\right)$ by (4.34) (right). Next, returning to (4.2) (with $u \equiv 0$ ), we can write by the above regularity

$$
A^{-3 / 4} \mathscr{A}_{\gamma} w_{t t}=\left[-A^{1 / 4} w+A^{-3 / 4} F\right] \in C\left([0, T] ; L_{2}(\Omega)\right) .
$$

Invoking the characterization (3.39) for $\tilde{H}_{-1}(\Omega)$, we conclude that (4.38) holds true if and only if $w_{t t} \in C\left([0, T] ; \tilde{H}_{-1}(\Omega)\right)$, and (4.35) is established.

Proposition 4.6 (well-posedness of (4.5) on $\mathscr{D}\left(\mathbb{A}_{0, \gamma}^{2}\right)$ ). With reference to problem (4.1) with $u \equiv 0$, let, via (4.10),

$$
\begin{gathered}
\left\{w_{0}, w_{1}\right\} \in \mathscr{D}\left(\mathbb{A}_{0, \gamma}^{2}\right)=\mathscr{D}\left(A^{1 / 2} \mathscr{A}_{\gamma}^{-1} A\right) \times \mathscr{D}\left(A^{3 / 4}\right), \text { equivalently } \\
A w_{0} \in \mathscr{H}^{\perp} ; w_{1} \in H^{3}(\Omega) \cap H_{0}^{2}(\Omega), \\
F \in L_{1}\left(0, T ;\left[H^{1}(\Omega) \cap \mathscr{H}^{\perp}\right]\right) .
\end{gathered}
$$

Then, continuously,

$$
\begin{aligned}
\left\{w, w_{t}\right\} \in C\left([0, T] ; \mathscr{D}\left(\mathbb{A}_{0, \gamma}^{2}\right)\right) & \Longleftrightarrow A w \in C\left([0, T] ; \mathscr{H}^{\perp}\right) \\
& \Longleftrightarrow w_{t} \in C\left([0, T] ; H^{3}(\Omega) \cap H_{0}^{2}(\Omega)\right),
\end{aligned}
$$

with regularity for $w_{t t}$ given below in (4.42) and (4.43). 
Proof. By Lemma 3.1(a3), we have that $\mathscr{A}_{\gamma}^{-1} F \in L_{1}\left(0, T ; \mathscr{D}\left(A^{3 / 4}\right)\right)$ if and only if $F \in L_{1}\left(0, T ;\left[H^{1}(\Omega) \cap \mathscr{H}^{\perp}\right]\right)$, which is hypothesis (4.40). Accordingly, then, $\left[0, \mathscr{A}_{\gamma}^{-1} F\right] \in L_{1}\left(0, T ; \mathscr{D}\left(\mathbb{A}_{0, \gamma}^{2}\right)\right)$, by (4.10), and thus (4.41) follows by (4.9) and (4.39). Moreover, returning to (4.2) with $u \equiv 0$, we get

$$
w_{t t}=-\mathscr{A}_{\gamma}^{-1} A w+\mathscr{A}_{\gamma}^{-1} F
$$

where by (4.41a) and the above remark

$$
\mathscr{A}_{\gamma}^{-1} A w \in C\left([0, T] ; \mathscr{A}_{\gamma}^{-1} \mathscr{H}^{\perp}\right) ; \quad \mathscr{A}_{\gamma}^{-1} F \in L_{1}\left(0, T ; \mathscr{D}\left(A^{3 / 4}\right)\right) .
$$

Proposition 4.7 (well-posedness of $(4.5)$ on $\left.\left[\mathscr{D}\left(\left(A_{0, \gamma}^{*}\right)^{2}\right)\right]^{\prime}\right)$. With reference to problem (4.1) with $u \equiv 0$, let, via (4.13),

$$
\begin{gathered}
\left\{w_{0}, w_{1}\right\} \in\left[\mathscr{D}\left(\left(\mathbb{A}_{0, \gamma}^{*}\right)^{2}\right)\right]^{\prime}=\tilde{L}_{2}(\Omega) \times \tilde{H}_{-1}(\Omega), \\
F \in L_{1}\left(0, T ;\left[\mathscr{D}\left(A^{3 / 4}\right)\right]^{\prime}\right) .
\end{gathered}
$$

Then, continuously,

$$
\left\{w, w_{t}\right\} \in C\left([0, T] ;\left[\mathscr{D}\left(\left(A_{0, \gamma}^{*}\right)^{2}\right)\right]^{\prime} \equiv \tilde{L}_{2}(\Omega) \times \tilde{H}_{-1}(\Omega)\right) .
$$

Proof. By (3.39), we have that $\mathscr{A}_{\gamma}^{-1} F \in L_{1}\left(0, T ; \tilde{H}_{-1}(\Omega)\right)$ if and only if $A^{-3 / 4} \mathscr{A}_{\gamma} \mathscr{A}_{\gamma}^{-1} F=A^{-3 / 4} F \in L_{1}\left(0, T ; L_{2}(\Omega)\right)$, which is guaranteed by assumption (4.45). Hence, $\left[0, \mathscr{A}_{\gamma}^{-1} F\right] \in L_{1}\left(0, T ;\left[\mathscr{D}\left(\left(\mathbb{A}_{0, \gamma}^{*}\right)^{2}\right)\right]^{\prime}\right) \equiv \tilde{L}_{2}(\Omega) \times \tilde{H}_{-1}(\Omega)$ by (4.13), or $\mathbb{A}_{0, \gamma}^{-2}\left[0, A_{\gamma}^{-1} F\right] \in L_{1}\left(0, T ; Y_{\gamma}\right)$. It follows, via (4.9), that

$$
\begin{aligned}
\mathbb{A}_{0, \gamma}^{-2}\left[\begin{array}{c}
w(t) \\
w_{t}(t)
\end{array}\right]= & e^{\mathbb{A}_{0, \gamma} t} \mathbb{A}_{0, \gamma}^{-2}\left[\begin{array}{c}
w_{0} \\
w_{1}
\end{array}\right] \\
& +\int_{0}^{t} e^{\mathbb{A}_{0, \gamma}(t-\tau)} \mathbb{A}_{0, \gamma}^{-2}\left[\begin{array}{c}
0 \\
\mathscr{A}_{\gamma}^{-1} F(\tau)
\end{array}\right] d \tau \in C\left([0, T] ; Y_{\gamma}\right),
\end{aligned}
$$

and (4.46) is proved via (4.47).

4.3. Interpolation results for $u \equiv 0$. We next provide some interpolation results based on those of Section 4.2. It will suffice to take zero initial conditions: $\left\{w_{0}, w_{1}\right\}=0$. The next result reproduces [21, Corollary 3.5, page 417] for $\left\{w, w_{t}\right\}$ but clarifies this result regarding $w_{t t}$.

Proposition 4.8 (interpolation between $\mathscr{D}\left(\mathbb{A}_{0, \gamma}\right)$ and $\left.Y_{\gamma}\right)$. With reference to problem (4.1) with $u \equiv 0$ and $w_{0}=w_{1}=0$, let $0 \leq \beta \leq 1$, and let 
Factor spaces and Kirchhoff equations

(a)

$$
\mathscr{A}_{\gamma}^{-1} F \in L_{1}\left(0, T ; \mathscr{D}\left(A^{1 / 2-\beta / 4}\right)\right)
$$

Then continuously,

$$
\begin{gathered}
w \in C\left([0, T] ; \mathscr{D}\left(A^{(3-\beta) / 4}\right)\right) ; \\
w_{t} \in C\left([0, T] ; \mathscr{D}\left(A^{(1 / 2-\beta / 4)}\right)\right) .
\end{gathered}
$$

(b) Assume (4.48) with $L_{2}$ in place of $L_{1}$. Then, continuously,

$$
w_{t t} \in L_{2}\left(0, T ; \mathscr{D}\left(A^{(1-\beta) / 4}\right)\right) \text {. }
$$

(c) We explicitly single out the special case $\beta=1 / 2$, in which case we obtain

$$
\begin{aligned}
& \mathscr{A}_{\gamma}^{-1} F \in L_{1}\left(0, T ; \mathscr{D}\left(A^{3 / 8}\right)\right) \Longrightarrow\left\{\begin{array}{l}
w \in C\left([0, T] ; \mathscr{D}\left(A^{5 / 8}\right)\right) ; \\
w_{t} \in C\left([0, T] ; \mathscr{D}\left(A^{3 / 8}\right)\right) ;
\end{array}\right. \\
& \mathscr{A}_{\gamma}^{-1} F \in L_{2}\left(0, T ; \mathscr{D}\left(A^{3 / 8}\right)\right) \Longrightarrow w_{t t} \in L_{2}\left(0, T ; \mathscr{D}\left(A^{1 / 8}\right)\right),
\end{aligned}
$$

continuously, where recalling (2.3) and [19, page 10] we have

$$
\begin{aligned}
\mathscr{D}\left(\mathscr{A}_{\gamma}^{1 / 4}\right) & =\mathscr{D}\left(\mathscr{A}^{1 / 4}\right)=\mathscr{D}\left(A^{1 / 8}\right)=\left[\mathscr{D}\left(A^{1 / 4}\right), L_{2}(\Omega)\right]_{1 / 2} \\
& =\left[H_{0}^{1}(\Omega), L_{2}(\Omega)\right]_{1 / 2}=H_{00}^{1 / 2}(\Omega), \\
\mathscr{D}\left(\mathscr{A}_{\gamma}^{3 / 4}\right) & =\mathscr{D}\left(\mathscr{A}^{3 / 4}\right)=\mathscr{D}\left(A^{3 / 8}\right)=\left[\mathscr{D}\left(A^{1 / 2}\right), \mathscr{D}\left(A^{1 / 4}\right)\right]_{1 / 2} \\
& =\left[H_{0}^{2}(\Omega), H_{0}^{1}(\Omega)\right]_{1 / 2}=H_{00}^{3 / 2}(\Omega), \\
\mathscr{D}\left(A^{5 / 8}\right) & =\left[\mathscr{D}\left(A^{3 / 4}\right), \mathscr{D}\left(A^{1 / 2}\right)\right]_{1 / 2} \\
& =\left[H^{3}(\Omega) \cap H_{0}^{2}(\Omega), H_{0}^{2}(\Omega)\right]_{1 / 2} .
\end{aligned}
$$

Proof. (a) We interpolate between

$$
\text { the case } \beta=0: \mathscr{A}_{\gamma}^{-1} F \in L_{1}\left(0, T ; \mathscr{D}\left(A^{1 / 2}\right)\right) \text {, }
$$

see (4.27) contained in Proposition 4.4,

and the case $\beta=1: \mathscr{A}_{\gamma}^{-1} F \in L_{1}\left(0, T ; \mathscr{D}\left(A^{1 / 4}\right)=\mathscr{D}\left(\mathscr{A}_{\gamma}^{1 / 2}\right)\right)$

(deduced from (4.19)) contained in Proposition 4.3,

to readily obtain (4.49) for $\left\{w, w_{t}\right\}$ from (4.28) and (4.20).

(b) Similarly to part (a), with $L_{2}$ replacing $L_{1}$, we readily obtain (4.50) from (4.29) and (4.22).

Then (4.49) and (4.50) specialize to (4.51), respectively, for $\beta=1 / 2$. 
Proposition 4.9 (interpolation between $Y_{\gamma}$ and $\left.\left[\mathscr{D}\left(\mathbb{A}_{0, \gamma}^{*}\right)\right]^{\prime}\right)$. With reference to problem (4.1) with $u \equiv 0$ and $w_{0}=w_{1}=0$, let $0 \leq \beta \leq 1$, and let

$$
A^{-1 / 2} F \in L_{1}\left(0, T ; \mathscr{D}\left(A^{(1-\beta) / 4}\right)\right) \text {. }
$$

Then, continuously,

$$
\begin{aligned}
& w \in C\left([0, T] ; \mathscr{D}\left(A^{1 / 2-\beta / 4}\right)\right) ; \\
& w_{t} \in C\left([0, T] ;\left[\mathscr{D}\left(A^{1 / 4}\right), \tilde{L}_{2}(\Omega)\right]_{\beta}\right) ;
\end{aligned}
$$

or

$$
\begin{gathered}
\mathscr{H}^{\perp} \ni \Pi w_{t} \in C\left([0, T] ;\left[\mathscr{D}\left(A^{1 / 4}\right), L_{2}(\Omega)\right]_{\beta}\right) ; \\
{\left[H_{0}^{1}(\Omega), L_{2}(\Omega)\right]_{\beta}=\left[\mathscr{D}\left(A^{1 / 4}\right), L_{2}(\Omega)\right]_{\beta}} \\
= \begin{cases}H_{0}^{1-\beta}(\Omega), & 0 \leq \beta \neq \frac{1}{2} \leq 1 ; \\
H_{00}^{1 / 2}(\Omega), & \beta=\frac{1}{2} .\end{cases}
\end{gathered}
$$

Proof. We interpolate between

the case $\beta=0: A^{-1 / 2} F \in L_{1}\left(0, T ; \mathscr{D}\left(A^{1 / 4}\right)\right) \Longleftrightarrow A^{-1 / 4} F \in L_{1}\left(0, T ; L_{2}(\Omega)\right)$, see (4.19) contained in Proposition 4.3;

and the case $\beta=1: A^{-1 / 2} F \in L_{1}\left(0, T ; L_{2}(\Omega)\right)$,

see (4.32), contained in Proposition 4.5,

to readily obtain (4.55) for $\left\{w, w_{t}\right\}$ from (4.20) and (4.33).

4.4. The case of point-control, $\operatorname{dim} \Omega=3$. We return to the point control problem of [21, Section 3], which consists of (4.1a), and (4.1b) with $w_{0}=$ $w_{1}=0, u \equiv 0, F=\delta(x) v(t)$, where $\delta(x)$ is the Dirac distribution at the origin assumed to be an interior point of $\Omega, \operatorname{dim} \Omega=3$; that is

$$
\begin{array}{ll}
w_{t t}-\gamma \Delta w_{t t}+\Delta^{2} w=\delta(x) v(t) & \text { in } Q ; \\
w(0, \cdot)=0, \quad w_{t}(0, \cdot)=0 & \text { in } \Omega \\
\left.w\right|_{\Sigma} \equiv 0,\left.\quad \frac{\partial w}{\partial v}\right|_{\Sigma} \equiv 0 & \text { in } \Sigma .
\end{array}
$$

The following result recovers the regularity of $\left\{w, w_{t}\right\}$, solution of (4.58), as given in [21, equations (3.13a-b)], but makes more precise [21, equation (3.13c)] regarding the regularity of $w_{t t}$. 
Theorem 4.10. Let $\operatorname{dim} \Omega=3$ and let $v \in L_{2}(0, T)$. Then the solution of problem (4.58) satisfies

$$
\left\{w, w_{t}\right\} \in C\left([0, T] ; H_{0}^{2}(\Omega) \times H_{0}^{1}(\Omega)\right) ; \quad w_{t t} \in L_{2}\left(0, T ; L_{2}(\Omega)\right)
$$

continuously.

Proof. For $\operatorname{dim} \Omega=3$, the Kirchhoff $h$-problem in [21, equations (3.22a-c)], is precisely problem (4.1) with $u \equiv 0$ and $F \in L_{2}\left(0, T ; H^{-1}(\Omega)\right)$ or $A^{-1 / 4} F \in$ $L_{2}\left(0, T ; L_{2}(\Omega)\right)$, see [21, equation (3.23a)]. Then, its regularity is covered by Proposition 4.3, (4.20) and (4.22). (Proposition 4.3 was observed already to be [21, Proposition 3.4] regarding $\left\{w, w_{t}\right\}$.) Thus, $\left\{h, h_{t}\right\} \in C\left([0, T] ; H_{0}^{2}(\Omega) \times\right.$ $H_{0}^{1}(\Omega)$ ), as asserted in [21, equations (3.24a-b)], while $h_{t t} \in L_{2}\left(0, T ; L_{2}(\Omega)\right)$, a refinement over [21, equation (3.24c)]. Finally, returning to the original variable $w=\psi \phi-h$ in the notation of [21], where $\left\{\phi, \phi_{t}, \phi_{t t}\right\}$ satisfy [21, equations $(2.17 \mathrm{a}-\mathrm{c})]$, we obtain (4.59), as desired.

\subsection{The non-homogeneous boundary case $u \neq 0$}

Proof of Theorem 1.1. In this section, which is complementary to the previous sections, we consider the mixed problem (4.1) with

$$
w_{0}=0, \quad w_{1}=0 ; \quad F \equiv 0 ; \quad u \in L_{2}\left(0, T ; L_{2}(\Gamma)\right),
$$

whose abstract model is given by (4.2). In Proposition 4.5, we have seen that if, say, $F \equiv 0, u \equiv 0$, and $w \in C\left([0, T] ; \mathscr{D}\left(A^{1 / 4}\right)\right)$, then $A^{-3 / 4} A_{\gamma} w_{t t}=-A^{1 / 4} w \in$ $C\left([0, T] ; L_{2}(\Omega)\right)$, and thus $w_{t t} \in C\left([0, T] ; \tilde{H}_{-1}(\Omega)\right)$ by (3.39). The next result complements Proposition 4.3 by considering the case where $u \in$ $L_{2}\left(0, T ; L_{2}(\Gamma)\right)$.

Theorem 4.11. Consider problem (4.1) subject to hypothesis (4.60). Then, continuously,

$$
\begin{aligned}
w & \in C\left([0, T] ; H_{0}^{1}(\Omega) \equiv \mathscr{D}\left(A^{1 / 4}\right)\right) ; \\
w_{t} & \in C\left([0, T] ; \tilde{L}_{2}(\Omega)\right) \\
w_{t t} & \in L_{2}\left(0, T ; \tilde{H}_{-1}(\Omega)\right)
\end{aligned}
$$

Proof. Conclusion (4.61) and (4.62) on $\left\{w, w_{t}\right\}$ follows by duality on the sharp trace regularity (4.21) of the corresponding homogeneous problem, due to [7, Chapter 5]. Here are the details, taken, for example, from [4, 22]. We return to the abstract model (4.2) of the mixed problem (4.1) under assumption (4.60), the corresponding solution is then written as

$$
\left[\begin{array}{c}
w(t) \\
w_{t}(t)
\end{array}\right]=(L u)(t)=\int_{0}^{t} e^{\mathbb{A}_{0, \gamma}(t-\tau)} \mathscr{B}_{c}\left[\begin{array}{c}
0 \\
u(\tau)
\end{array}\right] d \tau,
$$


where [22], [4, equations (3.34), (3.37)],

$$
\mathscr{B}_{c}\left[\begin{array}{l}
0 \\
u
\end{array}\right]=\left[\begin{array}{c}
0 \\
\mathscr{A}_{\gamma}^{-1} A G_{2} u
\end{array}\right], \quad G_{2}^{*} A f=-\left.\Delta f\right|_{\Gamma}, \quad f \in \mathscr{D}(A) .
$$

Let (in the notation of [4, equations (5.2.2), (5.2.4)]),

$$
\left[\mathscr{D}\left(\mathbb{A}_{0, \gamma}^{*}\right)\right]^{\prime}=\mathscr{D}\left(A^{1 / 4}\right) \times \tilde{L}_{2}(\Omega) \equiv H_{0}^{1}(\Omega) \times \tilde{L}_{2}(\Omega)
$$

(norm equivalence) recalling critically (4.12), hence Section 2. If $L_{T} u=$ $(L u)(T)$, and $x=\left[x_{1}, x_{2}\right] \in\left[\mathscr{D}\left(\mathbb{A}_{0, \gamma}^{*}\right)\right]^{\prime}$, then [4, equation (5.2.6)],

$$
\left(L_{T}\left[\begin{array}{l}
0 \\
u
\end{array}\right],\left[\begin{array}{l}
x_{1} \\
x_{2}
\end{array}\right]\right)_{\left[\mathscr{D}\left(\mathbb{A}_{0, \gamma}^{*}\right)\right]^{\prime}}=\left(\left[\begin{array}{l}
0 \\
u
\end{array}\right], L_{T}^{\#}\left[\begin{array}{l}
x_{1} \\
x_{2}
\end{array}\right]\right)_{L_{2}(\Sigma) \times L_{2}(\Sigma)},
$$

where [4, equation (5.2.8)],

$$
\left(L_{T}^{\#}\left[\begin{array}{l}
x_{1} \\
x_{2}
\end{array}\right]\right)(t)=\left[\begin{array}{c}
0 \\
\left.\Delta \psi\left(T-t ; y_{0}\right)\right|_{\Gamma}
\end{array}\right],
$$

and where [4],

$$
\begin{gathered}
{\left[\begin{array}{c}
\psi\left(T-t ; y_{0}\right) \\
-\psi_{t}\left(T-t ; y_{0}\right)
\end{array}\right]=e^{\mathbb{A}_{0, \gamma}^{*}(T-t)} y_{0} ;} \\
y_{0}=\left[\begin{array}{c}
\psi_{0} \\
-\psi_{1}
\end{array}\right]=\mathbb{A}_{0, \gamma}^{-1}\left[\begin{array}{c}
x_{1} \\
x_{2}
\end{array}\right] \in Y_{\gamma} \equiv \mathscr{D}\left(A^{1 / 2}\right) \times \mathscr{D}\left(A_{\gamma}^{1 / 2}\right) \equiv H_{0}^{2}(\Omega) \times H_{0}^{1}(\Omega)
\end{gathered}
$$

is the solution of the homogeneous problem

$$
\begin{array}{ll}
\psi_{t t}-\gamma \Delta \psi_{t t}+\Delta^{2} \psi \equiv 0 & \text { in } Q ; \\
\psi(0, \cdot)=\psi_{0}, \quad \psi_{t}(0, \cdot)=\psi_{1} & \text { in } \Omega ; \\
\left.\psi\right|_{\Sigma} \equiv 0,\left.\quad \frac{\partial \psi}{\partial \nu}\right|_{\Sigma} \equiv 0 & \text { in } \Sigma .
\end{array}
$$

By [7, equation (2.24), page 123], reported in (4.21) (with $F \equiv 0$ ), we have the following trace result for problem (4.71):

$$
\left\{\psi_{0}, \psi_{1}\right\} \in H_{0}^{2}(\Omega) \times\left. H_{0}^{1}(\Omega) \longrightarrow \Delta \psi\right|_{\Gamma} \in L_{2}\left(0, T ; L_{2}(\Gamma)\right)
$$

continuously. Equivalently, via (4.68) and (4.70),

$$
L_{T}^{\#} \text { : continuous }\left[\mathscr{D}\left(\mathbb{A}_{0, \gamma}^{*}\right)\right]^{\prime} \longrightarrow L_{2}\left(0, T ; L_{2}(\Gamma)\right) .
$$


Then (4.72) is, in turn, equivalent to [12]

$$
\begin{aligned}
L \text { : continuous } & L_{2}\left(0, T ; L_{2}(\Gamma)\right) \\
& \longrightarrow(L u)=\left\{w, w_{t}\right\} \in C\left([0, T] ; H_{0}^{1}(\Omega) \times \tilde{L}_{2}(\Omega)\right),
\end{aligned}
$$

and (4.61) and (4.62) are established by duality on (4.72).

We now establish (4.63). We return to the abstract model (4.2), which we rewrite as

$$
A^{-3 / 4} \mathscr{A}_{\gamma} w_{t t}=-A^{1 / 4} w+A^{-1 / 8+\epsilon}\left(A^{3 / 8-\epsilon} G_{2} u\right) \in L_{2}\left(0, T ; L_{2}(\Omega)\right),
$$

where the regularity noted in (4.75) follows from $A^{1 / 4} w \in C\left([0, T] ; L_{2}(\Omega)\right)$ by (4.61), as well as from $A^{3 / 8-\epsilon} G_{2} u \in L_{2}\left(0, T ; L_{2}(\Omega)\right)$ by (4.4b) on $G_{2}$ and (4.60) on $u$. Thus, as usual via the characterization (3.39), we see that (4.75) says that $w_{t t} \in L_{2}\left(0, T ; \tilde{H}_{-1}(\Omega)\right)$, as claimed in (4.63). (The above argument shows that, in the present circumstances, the term $A w$ is the critical one, while $A G_{2} u$ is subordinated to it, in model (4.2).)

\section{Implications on regularity of mixed Kirchhoff thermoelastic plate equa- tions with clamped BC: proof of Theorems 1.2 and 1.3}

5.1. Proof of Theorem 1.2. In this subsection we let $\Omega$ be an open bounded domain in $\mathbb{R}^{n}$, for any positive integer $n$, with smooth boundary $\Gamma$. On $\Omega$ we consider the following thermoelastic mixed problem in the unknown $\{w(t, x)$, $\theta(t, x)\}$ :

$$
\begin{array}{ll}
w_{t t}-\gamma \Delta w_{t t}+\Delta^{2} w+\Delta \theta=0 & \text { in }(0, T] \times \Omega=Q ; \\
\theta_{t}-\Delta \theta-\Delta w_{t}=0 & \text { in } Q ; \\
w(0, \cdot)=w_{0} ; \quad w_{t}(0, \cdot)=w_{1} ; \quad \theta(0, \cdot)=\theta_{0} & \text { in } \Omega ; \\
\left.w\right|_{\Sigma} \equiv 0 ;\left.\quad \frac{\partial w}{\partial v}\right|_{\Sigma}=u ;\left.\quad \theta\right|_{\Sigma} \equiv 0 & \text { in }(0, T] \times \Gamma \equiv \Sigma,
\end{array}
$$

where, for our present purposes, it will suffice to take

$$
w_{0}=0, \quad w_{1}=0, \quad \theta_{0}=0 ; \quad u \in L_{2}\left(0, T ; L_{2}(\Gamma)\right) .
$$

As in the elastic case of Section 4, in the present thermoelastic case we will take the constant $\gamma>0$ throughout. Our goal is to establish the following regularity result, which is sharp in $\left\{w, w_{t}\right\}$. It is a restatement of Theorem 1.2.

THEOREM 5.1. With reference to the mixed problem (5.1) with $\gamma>0$ and zero initial conditions as in (5.2), the following regularity results hold true, where 
$\mathscr{D}\left(A^{1 / 4}\right) \equiv H_{0}^{1}(\Omega)$ (norm equivalence): the map

$$
\begin{aligned}
& u \in L_{2}\left(0, T ; L_{2}(\Gamma)\right) \Longrightarrow\left\{w, w_{t}\right\} \in C\left([0, T] ; \mathscr{D}\left(A^{1 / 4}\right) \times \tilde{L}_{2}(\Omega)\right) ; \\
& \Longrightarrow\left[w_{t t}-\frac{1}{\gamma} \theta\right] \in L_{2}\left(0, T ; \tilde{H}_{-1}(\Omega)\right) ; \\
& \Longrightarrow \theta \in L_{p}\left(0, T ; H^{-1}(\Omega)\right) \cap C\left([0, T] ; H^{-1-\epsilon}(\Omega)\right), \\
& 1<p<\infty ; \forall \epsilon>0,
\end{aligned}
$$

is continuous. However, in addition, we have

$$
\begin{aligned}
& \theta \in C\left([0, T] ; L_{2}(\Omega)\right), \text { and } w_{t t} \in L_{2}\left(0, T ; \tilde{H}_{-1}(\Omega)\right), \\
& \text { but not continuously in } u \in L_{2}\left(0, T ; L_{2}(\Gamma)\right)
\end{aligned}
$$

(that is, the closed graph theorem does not apply to the maps $u \rightarrow \theta$ or $u \rightarrow w_{t t}$ in (5.6)). More precisely, regarding $\theta$, we have

$$
\theta(t)=-w_{t}(t)+\theta_{1, a}(t)+\theta_{1, b}(t)
$$

where $w_{t}$ satisfies (5.3), and

$$
\begin{aligned}
& \theta_{1, b}(t)=\frac{1}{\gamma} \int_{0}^{t} e^{-\mathscr{A}(t-\tau)} \theta(\tau) d \tau \in C\left([0, T] ; L_{2}(\Omega)\right) \\
& \text { continuously in } u \in L_{2}\left(0, T ; L_{2}(\Gamma)\right) ;
\end{aligned}
$$

while

$$
\begin{aligned}
\theta_{1, a}(t) & =\int_{0}^{t} e^{-\mathscr{A}(t-\tau)}\left[w_{t t}(\tau)-\frac{1}{\gamma} \theta(\tau)\right] d \tau \\
& \in C\left([0, T] ; L_{2}(\Omega)\right) \text { however, not continuously in } u \\
& \in L_{2}\left(0, T ; L_{2}(\Gamma)\right)
\end{aligned}
$$

Proof of Theorem 5.1. The mechanical regularity (5.3) for $\left\{w, w_{t}\right\}$ was established in [22, Theorem 4.1], and coincides with the mechanical regularity (4.61) and (4.62) in the elastic case of Theorem 4.11. We will repeat a sketch of the argument for completeness, following [22] or [4].

Step 1. We start with the dual $\{\phi, \eta\}$-thermoelastic problem:

$$
\begin{array}{ll}
\phi_{t t}-\gamma \Delta \phi_{t t}+\Delta^{2} \phi-\Delta \eta=0 & \text { in } Q=(0, T] \times \Omega ; \\
\eta_{t}-\Delta \eta+\Delta \phi_{t}=0 & \text { in } Q ; \\
\phi(0, \cdot)=\phi_{0}, \quad \phi_{t}(0, \cdot)=\phi_{1}, \quad \eta(0, \cdot)=\eta_{0} & \text { in } \Omega ; \\
\left.\phi\right|_{\Sigma} \equiv 0,\left.\quad \frac{\partial \phi}{\partial v}\right|_{\Sigma} \equiv 0,\left.\quad \eta\right|_{\Sigma} \equiv 0 & \text { in } \Sigma=(0, T] \times \Gamma,
\end{array}
$$


with initial conditions,

$$
\left\{\phi_{0},-\phi_{1}, \eta_{0}\right\} \in Y_{\gamma} \equiv H_{0}^{2}(\Omega) \times H_{0}^{1}(\Omega) \times L_{2}(\Omega)
$$

Thus, the solution $\left\{\phi(t),-\phi_{t}(t), \eta(t)\right\}=e^{\mathbb{A}_{\gamma}^{*} t}\left[\phi_{0},-\phi_{1}, \eta_{0}\right]$ is given by the adjoint semigroup $e^{\mathbb{A}_{\gamma}^{*} t}$ on $Y_{\gamma}$ to the one $e^{\mathbb{A}_{\gamma} t}$ claimed (by Lumer-Phillips theorem) in [22, below (4.17)], [4, equation (2.10)], and so forth. Thus, its a priori regularity is

$$
\begin{gathered}
\left\{\phi,-\phi_{t}, \eta\right\} \in C\left([0, T] ; H_{0}^{2}(\Omega) \times H_{0}^{1}(\Omega) \times L_{2}(\Omega)\right) ; \\
\eta \in L_{2}\left(0, T ; H_{0}^{1}(\Omega)\right), \quad \Delta \eta \in L_{2}\left(0, T ; H^{-1}(\Omega)\right),
\end{gathered}
$$

using also the usual dissipativity argument for $\eta$ [18]. Next, we rewrite problem (5.9) in the following way:

$$
\begin{gathered}
\phi_{t t}-\gamma \Delta \phi_{t t}+\Delta^{2} \phi=\Delta \eta \in L_{2}\left(0, T ; H^{-1}(\Omega)\right) ; \\
\left.\phi\right|_{\Sigma} \equiv 0,\left.\quad \frac{\partial \phi}{\partial v}\right|_{\Sigma} \equiv 0 .
\end{gathered}
$$

Step 2. To problem (5.12) we apply the same energy method proof in [7, Chapter 5 or Chapter 6] by use of the multiplier $m \cdot \nabla \phi,\left.m\right|_{\Gamma}=v$, it yields the following sharp trace regularity:

$$
\left\{\phi_{0}, \phi_{1}\right\} \in H_{0}^{2}(\Omega) \times\left. H_{0}^{1}(\Omega) \Longrightarrow \Delta \phi\right|_{\Gamma} \in L_{2}\left(0, T ; L_{2}(\Gamma)\right),
$$

(where, of course, $\eta_{0} \in L_{2}(\Omega)$ as well) since $\int_{Q}(m \cdot \nabla \phi)(\Delta \eta) d Q$ is finite by use of (5.11), $f=m \cdot \nabla \phi \in C\left([0, T] ; H_{0}^{1}(\Omega)\right)$ since $\left.f\right|_{\Gamma}=\left.m \cdot \nabla \phi\right|_{\Gamma}=$ $\left.(\partial \phi / \partial v)\right|_{\Gamma}=0$. Details are given, for example, in [4, equations (3.97)-(3.99), page 129; and also equations (C.47)-(C.49), page 206].

Step 3. A duality argument, given in details in [22, Section 5, Step 3] in [4, Step 1, page 206], then shows the following preliminary result: for the mixed problem (5.1) and (5.2), the map

$$
u \in L_{2}(\Sigma) \longrightarrow\left\{\begin{array}{l}
\left\{w, w_{t}\right\} \in C\left([0, T] ; H_{0}^{1}(\Omega) \times \tilde{L}_{2}(\Omega)\right) \\
\theta \in C\left([0, T] ;[\mathscr{D}(\mathscr{A})]^{\prime}\right)
\end{array}\right.
$$

is continuous. This map is optimal for $\left\{w, w_{t}\right\}$. The space $\tilde{L}_{2}(\Omega)$ described in Section 2 arises at this point, in connection with the second component space, as dual of $\mathscr{D}\left(A^{1 / 2}\right)$ with respect to $\mathscr{D}\left(\mathscr{A}_{\gamma}^{1 / 2}\right)$ as a pivot space. So far, we have reproduced results of $[4,22]$ for the mixed problem (5.1) and (5.2). Thus, we have established (5.3) for $\left\{w, w_{t}\right\}$. 
Step 4 (proof of (5.5)). We next boost the regularity of $\theta$ (over (5.14)) to read the map

$$
\begin{array}{r}
u \in L_{2}\left(0, T ; L_{2}(\Gamma)\right) \longrightarrow \theta \in L_{p}\left(0, T ; H^{-1}(\Omega)\right) \cap C\left([0, T] ; H^{-1-\epsilon}(\Omega)\right), \\
1<p<\infty ; \forall \epsilon>0,
\end{array}
$$

is continuous. To establish (5.15), we return to (5.1b) and integrate by parts to obtain via $\left.w_{t}\right|_{\Sigma} \equiv 0$ and (2.2)

$$
\begin{aligned}
\theta(t) & =\int_{0}^{t} e^{-\mathscr{A}(t-\tau)} \Delta w_{t}(\tau) d \tau=-\int_{0}^{t} e^{-\mathscr{A}(t-\tau)} \mathscr{A} w_{t}(\tau) d \tau \\
& =-\mathscr{A}^{1 / 2} \mathscr{A}^{1 / 2} w(t)+\mathscr{A}^{1 / 2+\epsilon / 2} \int_{0}^{t} \mathscr{A}^{1-\epsilon / 2} e^{-\mathscr{A}(t-\tau)} \mathscr{A}^{1 / 2} w(\tau) d \tau \\
& \in L_{p}\left(0, T ; H^{-1}(\Omega)\right) \cap C\left([0, T] ; H^{-1-\epsilon}(\Omega)\right),
\end{aligned}
$$

for all $1<p<\infty$; for all $\epsilon>0$, since $w_{0}=0$ by (5.2). The regularity in (5.18) is obtained by using the regularity (5.3) for $w$, along with the following two well-known results for analytic semigroups: the map

$$
f \longrightarrow \int_{0}^{t} e^{-\mathscr{A}(t-\tau)} f(\tau) d \tau
$$

is continuous as follows:

$$
\begin{gathered}
L_{p}\left(0, T ; L_{2}(\Omega)\right) \longrightarrow L_{p}(0, T ; \mathscr{D}(\mathscr{A})), \quad \forall 1<p<\infty ; \\
L_{\infty}\left(0, T ; L_{2}(\Omega)\right) \longrightarrow C\left([0, T] ; \mathscr{D}\left(\mathscr{A}^{1-\epsilon}\right)\right), \quad \forall \epsilon>0,
\end{gathered}
$$

see [2] for (5.20), [16, page 4]. Thus, (5.15) is proved via (5.18).

Remark 5.2. The weaker result, over (5.6), that

$$
\begin{aligned}
& \theta \in L_{p}\left(0, T ; L_{2}(\Omega)\right) \cap C\left([0, T] ; H^{-\epsilon}(\Omega)\right), \quad \forall 1<p<\infty ; \forall \epsilon>0 ; \\
& \text { however, not continuously in } u \in L_{2}\left(0, T ; L_{2}(\Gamma)\right),
\end{aligned}
$$

follows at once from (5.16), via (5.3) on $w_{t},(2.49)$ and (5.20).

Step 5 (proof of (5.4)). The abstract model of the mixed problem (5.1) is given by $[4,22]$,

$$
\mathscr{A}_{\gamma} w_{t t}=-A w+A G_{2} u+\mathscr{A} \theta=-A w+A G_{2} u+\frac{1}{\gamma} \mathscr{A}_{\gamma} \theta-\frac{1}{\gamma} \theta
$$


(compare with (4.2)), from which we obtain

$$
\begin{aligned}
A^{-3 / 4} \mathscr{A}_{\gamma}\left[w_{t t}-\frac{1}{\gamma} \theta\right] & =-A^{1 / 4} w+A^{-1 / 8+\epsilon}\left(A^{3 / 8-\epsilon} G_{2} u\right)-\frac{1}{\gamma} A^{-3 / 4} \theta \\
& \in L_{2}\left(0, T ; L_{2}(\Omega)\right),
\end{aligned}
$$

continuously in $u \in L_{2}\left(0, T ; L_{2}(\Gamma)\right)$. The key regularity noted in $(5.23 \mathrm{~b})$ is obtained as follows: first, $A^{1 / 4} w \in C\left([0, T] ; L_{2}(\Omega)\right)$ by (5.3); next, $A^{3 / 8-\epsilon} G_{2} u$ $\in L_{2}\left(0, T ; L_{2}(\Omega)\right)$ by $(4.4 \mathrm{~b})$ and $u \in L_{2}\left(0, T ; L_{2}(\Gamma)\right)$ (just as in (4.75)); finally $A^{-3 / 4} \theta \in L_{2}\left(0, T ; \mathscr{D}\left(A^{1 / 2}\right)\right)$ by (5.5) with $p=2$, and $\mathscr{D}\left(A^{1 / 4}\right)=H_{0}^{1}(\Omega)$, see (2.3). Hence, the regularity (5.23b) is established. Then, as usual, via the characterization (3.39), we see that (5.23) says that $\left[w_{t t}-(1 / \gamma) \theta\right] \in$ $L_{2}\left(0, T ; \tilde{H}_{-1}(\Omega)\right)$, continuously in $u \in L_{2}\left(0, T ; L_{2}(\Gamma)\right)$, as claimed in (5.4). Step 6 (proof of (5.6) for $\theta$ ). We return to $(5.1 \mathrm{~b})$, which we rewrite as

$$
\left(\theta+w_{t}\right)_{t}=\Delta\left(\theta+w_{t}\right)+w_{t t},
$$

and hence, by (2.2),

$$
(\theta+w)_{t}=-\mathscr{A}\left(\theta+w_{t}\right)+w_{t t}
$$

because of the homogeneous BC (5.1d) for $w$ and $\theta$. Solving (5.24b), we obtain

$$
\theta(t)=-w_{t}(t)+\theta_{1}(t)
$$

where we will show that

$$
\theta_{1}(t)=\int_{0}^{t} e^{-\mathscr{A}(t-\tau)} w_{t t}(\tau) d \tau \in C\left([0, T] ; L_{2}(\Omega)\right),
$$

however, not continuously in $u \in L_{2}\left(0, T ; L_{2}(\Gamma)\right)$. We rewrite $\theta_{1}(t)$ as

$$
\begin{aligned}
\theta_{1}(t) & =\theta_{1, a}(t)+\theta_{1, b}(t) \\
\theta_{1, a}(t) & =\int_{0}^{t} e^{-\mathscr{A}(t-\tau)}\left[w_{t t}(\tau)-\frac{1}{\gamma} \theta(\tau)\right] d \tau ; \\
\theta_{1, b}(t) & =\frac{1}{\gamma} \int_{0}^{t} e^{-\mathscr{A}(t-\tau)} \theta(\tau) d \tau
\end{aligned}
$$

where, by (5.5) with $p=2$, and $H^{-1}(\Omega)=\left[\mathscr{D}\left(\mathscr{A}^{1 / 2}\right)\right]^{\prime}$, that is, with $\mathscr{A}^{-1 / 2} \theta \in$ $L_{2}\left(0, T ; L_{2}(\Omega)\right)$, continuously in $u \in L_{2}\left(0, T ; L_{2}(\Gamma)\right)$, we have

$$
\begin{aligned}
& \gamma \theta_{1, b}(t)=\int_{0}^{t} \mathscr{A}^{1 / 2} e^{-\mathscr{A}(t-\tau)} \mathscr{A}^{-1 / 2} \theta(\tau) d \tau \in C\left([0, T] ; L_{2}(\Omega)\right) \\
& \text { continuously in } u \in L_{2}\left(0, T ; L_{2}(\Gamma)\right) .
\end{aligned}
$$


Moreover, by (5.4), $\left[w_{t t}-\frac{1}{\gamma} \theta\right] \in L_{2}\left(0, T ; \tilde{H}_{-1}(\Omega)\right)$ continuously in $u \in$ $L_{2}\left(0, T ; L_{2}(\Gamma)\right)$; and since $\tilde{H}_{-1}(\Omega) \equiv\left[H^{1}(\Omega)\right]^{\prime} / \mathbb{H}$ by (3.38), we also have

$$
\begin{aligned}
& {\left[w_{t t}-\frac{1}{\gamma} \theta\right] \in L_{2}\left(0, T ;\left[H^{1}(\Omega)\right]^{\prime}\right) \text { however, not continuously }} \\
& \text { in } u \in L_{2}\left(0, T ; L_{2}(\Gamma)\right) .
\end{aligned}
$$

Hence, we obtain a fortiori that

$$
\left[w_{t t}-\frac{1}{\gamma} \theta\right] \in L_{2}\left(0, T ; H^{-1}(\Omega)\right) \text {, or } \mathscr{A}^{-1 / 2}\left[w_{t t}-\frac{1}{\gamma} \theta\right] \in L_{2}\left(0, T ; L_{2}(\Omega)\right) \text {, }
$$

however, not continuously in $u \in L_{2}\left(0, T ; L_{2}(\Gamma)\right)$.

It then follows from (5.29) and (5.27) that

$$
\theta_{1, a}(t)=\int_{0}^{t} \mathscr{A}^{1 / 2} e^{-\mathscr{A}(t-\tau)} \mathscr{A}^{-1 / 2}\left[w_{t t}(\tau)-\frac{1}{\gamma} \theta(\tau)\right] d \tau \in C\left([0, T] ; L_{2}(\Omega)\right),
$$

however, not continuously in $u \in L_{2}\left(0, T ; L_{2}(\Gamma)\right)$.

Then (5.28) and (5.31), used in (5.26) and (5.25), prove (5.6) for $\theta$, as desired; in fact, prove precisely (5.7) and (5.8).

Step 7 (proof of (5.6) for $w_{t t}$ ). We return to (5.22) (left form) and obtain

$$
\begin{aligned}
& A^{-3 / 4} \mathscr{A}_{\gamma} w_{t t}=-A^{1 / 4} w+A^{-1 / 8+\epsilon}\left(A^{-3 / 8-\epsilon} G_{2} u\right)+A^{-1 / 4}\left(A^{-1 / 2} \mathscr{A}\right) \theta \\
& \in L_{2}\left(0, T ; L_{2}(\Omega)\right), \text { however, not continuously in } u \in L_{2}\left(0, T ; L_{2}(\Gamma)\right) .
\end{aligned}
$$

The new term, over the analysis below (5.23b), is the last one in (5.32), where now $\theta$ satisfies (5.21) with $p=2$ (which now suffices without invoking (5.6)). Since $A^{-1 / 2} \mathscr{A}$ has a bounded extension on $L_{2}(\Omega)$ by (2.7), we conclude that

$$
\begin{aligned}
& A^{-1 / 4}\left(A^{-1 / 2} \mathscr{A}\right) \theta \in C\left([0, T] ; \mathscr{D}\left(A^{1 / 4}\right)=H_{0}^{1}(\Omega)\right), \text { however, not } \\
& \text { continuously in } u \in L_{2}\left(0, T ; L_{2}(\Gamma)\right) .
\end{aligned}
$$

Then the regularity in (5.32) is established by (5.33). The usual characterization (3.39) then yields that

$$
w_{t t} \in L_{2}\left(0, T ; \tilde{H}_{-1}(\Omega)\right) \text { however, not continuously in } u \in L_{2}\left(0, T ; L_{2}(\Gamma)\right) \text {. }
$$

The proof of Theorem 5.1 is complete. 
482 Factor spaces and Kirchhoff equations

5.2. Theorem 1.3: a sharp regularity result for $\theta_{1}(t)$ in $\mathbf{( 5 . 2 5 b )}$. We return to $\theta_{1}(t)$ in (5.25b) and prove the following result which complements, but does not replace, the regularity result in (5.6), (5.7), and (5.8). A restatement of Theorem 1.3 follows.

THEOREM 5.3. With reference to problem (5.1) and $\theta_{1}$ in (5.25b), the map $u \in L_{2}\left(0, T ; L_{2}(\Gamma)\right) \longrightarrow \theta_{1}(t)=\int_{0}^{t} e^{-\mathscr{A}(t-\tau)} w_{t t}(\tau) d \tau \in C\left([0, T] ;\left[H_{00}^{1 / 2}(\Omega)\right]^{\prime}\right)$,

is continuous, where $H_{00}^{1 / 2}(\Omega)=\mathscr{D}\left(\mathscr{A}^{1 / 4}\right)$ (see [19, page 66]). Thus, $\theta(t)=$ $-w_{t}(t)+\theta_{1}(t)$, where $w_{t}$ satisfies $(5.3): w_{t} \in C\left([0, T] ; \tilde{L}_{2}(\Omega)\right)$ continuously in $u \in L_{2}\left(0, T ; L_{2}(\Gamma)\right)$.

Sketch of proof. Here we may only give a sketch, which will highlight the main new key points. A full account will be given elsewhere [10], using also [15]. Step 1. Let $z \in H_{00}^{1 / 2}(\Omega)=\mathscr{D}\left(\mathscr{A}^{1 / 4}\right)$. Define

$$
v(t)=\mathscr{A}^{-1} e^{-\mathscr{A} t} z=\mathscr{A}^{-7 / 4} \mathscr{A}^{1 / 2} e^{-\mathscr{A} t} \mathscr{A}^{1 / 4} z \in L_{2}\left(0, T ; \mathscr{D}\left(\mathscr{A}^{7 / 4}\right)\right)
$$

Then, the following (parabolic theory) results hold true, both continuously in $z \in \mathscr{D}\left(\mathscr{A}^{1 / 4}\right)$ :

(i)

$$
v \in H^{7 / 2,7 / 4}(Q) \equiv L_{2}\left(0, T ; H^{7 / 2}(\Omega)\right) \cap H^{7 / 4}\left(0, T ; L_{2}(\Omega)\right) ;
$$

(ii) as a consequence of (5.37) and trace theory [19, Vol. 2, page 9], we have

$$
\frac{\partial v}{\partial v} \in H^{2,1}(\Sigma) \equiv L_{2}\left(0, T ; H^{2}(\Gamma)\right) \cap H^{1}\left(0, T ; L_{2}(\Gamma)\right) .
$$

Step 2. We return to (5.1a) and rewrite it, via (2.2) and (5.2), as

$$
\mathscr{A}_{\gamma} w_{t t}+\Delta^{2} w-\mathscr{A} \theta=0 ; \quad \text { or } \quad w_{t t}=-\mathscr{A}_{\gamma}^{-1} \Delta^{2} w+\mathscr{A}_{\gamma}^{-1} \mathscr{A} \theta
$$

(compare with (5.22)). Then, by (5.39), the critical term in the definition of $\theta_{1}(t)$ in $(5.35)$ is

$$
\tilde{\theta}(t) \equiv \int_{0}^{t} e^{-\mathscr{A}(t-\tau)} \mathscr{A}^{-1} \Delta^{2} w(\tau) d \tau .
$$

Our task is now reduced to show that the map

$$
\begin{aligned}
& u \in L_{2}\left(0, T ; L_{2}(\Gamma)\right) \longrightarrow \tilde{\theta} \in C\left([0, T] ;\left[H_{00}^{1 / 2}(\Omega)\right]^{\prime}\right) \text {, is continuous, } \\
& \text { where } H_{00}^{1 / 2}(\Omega)=\mathscr{D}\left(\mathscr{A}^{1 / 4}\right)
\end{aligned}
$$


To this end, we let at first $t$ be arbitrary but fixed in $(0, T], z \in H_{00}^{1 / 2}(\Omega)$ and $v$ defined by (5.36). Let $($,$) and \langle$,$\rangle denote the L_{2}(\Omega)$-and $L_{2}(\Gamma)$-inner products, respectively. By (5.40) and (5.36), we consider

$$
\begin{aligned}
(\tilde{\theta}(t), z) & =\int_{0}^{t}\left(e^{-\mathscr{A}(t-\tau)} \mathscr{A}^{-1} \Delta^{2} w(\tau), z\right) d \tau \\
(\operatorname{by}(5.36)) & =\int_{0}^{t}\left(\Delta^{2} w(\tau), v(t-\tau)\right) d \tau .
\end{aligned}
$$

Applying Green's second theorem to (5.42), we obtain

$$
\begin{aligned}
(\tilde{\theta}(t), z)= & \int_{0}^{t}(\Delta w(\tau), \Delta v(t-\tau)) d \tau \\
& +\int_{0}^{t}\left\langle\frac{\partial \Delta w(\tau)}{\partial v}, v(t-\tau)\right\rangle d \tau-\int_{0}^{t}\left\langle\Delta w(\tau), \frac{\partial v(t-\tau)}{\partial v}\right\rangle d \tau
\end{aligned}
$$

where $\left.v\right|_{\Gamma}=0$ by (5.36), and so the second integral term on $\Gamma$ in (5.43) vanishes. Moreover, we have, continuously in $u \in L_{2}\left(0, T ; L_{2}(\Gamma)\right)$

$$
\begin{aligned}
\Delta w \in C\left([0, T] ; H^{-1}(\Omega) \equiv\left[H_{0}^{1}(\Omega)\right]^{\prime}\right), \text { by }(5.3) ; \\
-\Delta v=\mathscr{A} v=v_{t} \in L_{2}\left(0, T ; \mathscr{D}\left(\mathscr{A}^{3 / 4}\right)\right) \equiv L_{2}\left(0, T ; H_{00}^{3 / 2}(\Omega)\right) \\
\subset L_{2}\left(0, T ; H_{0}^{3 / 2}(\Omega)\right) \subset L_{2}\left(0, T ; H_{0}^{1}(\Omega)\right),
\end{aligned}
$$

by (5.36), and by [19, page 66]:

$$
\mathscr{D}\left(\mathscr{A}^{3 / 4}\right) \equiv H_{00}^{3 / 2}(\Omega) \subset H_{0}^{3 / 2}(\Omega) .
$$

Thus, (5.44) and (5.46) imply that the first term on the right-hand side of (5.43) is well defined continuously in $u \in L_{2}\left(0, T ; L_{2}(\Gamma)\right)$. We use this fact, along with (5.38) and take, now, $z \in L_{1}\left(0, T ; H_{00}^{1 / 2}(\Omega)\right)$ in (5.43), to establish (5.41) and hence conclude the following lemma.

LEMMA 5.4. With reference to $\tilde{\theta}$ and $\theta_{1}$ in (5.40) and (5.35),

(i) the map

$u \in L_{2}\left(0, T ; L_{2}(\Gamma)\right) \longrightarrow \tilde{\theta} \in C\left([0, T] ;\left[H_{00}^{1 / 2}(\Omega)\right]^{\prime}\right)$ is continuous

and hence, as a consequence,

(ii) the map

$u \in L_{2}\left(0, T ; L_{2}(\Gamma)\right) \longrightarrow \theta_{1} \in C\left([0, T] ;\left[H_{00}^{1 / 2}(\Omega)\right]^{\prime}\right)$ is continuous 
provided that, in both cases, the solution $w$ to the mixed problem (5.1) satisfies the following trace regularity: the map

$$
\left.u \in L_{2}\left(0, T ; L_{2}(\Gamma)\right) \longrightarrow \Delta w\right|_{\Sigma} \in H^{-2,-1}(\Sigma) \text { is continuous }
$$

Step 3. The next result shows that the desired trace regularity (5.50) for problem (5.1) is, a fortiori, true, and hence (5.48) and (5.49) are established.

THEOREM 5.5. With reference to the mixed problem (5.1) the map

$$
\left.u \in L_{2}\left(0, T ; L_{2}(\Gamma)\right) \longrightarrow \Delta w\right|_{\Sigma} \in H^{-1,-1}(\Sigma) \text { is continuous. }
$$

Thus, a fortiori, the regularity properties (5.48) and (5.49) hold true.

It remains to sketch a proof of Theorem 5.5. The main points will be given in the steps below.

Step 4 (orientation). We rewrite the mixed problem (5.1) as

$$
\begin{gathered}
w_{t t}-\gamma \Delta w_{t t}+\Delta^{2} w-\Delta w_{t}=-\theta_{t} ; \\
w(0, \cdot)=0, \quad w_{t}(0, \cdot)=0, \quad \theta(0, \cdot)=0 ; \\
\left.w\right|_{\Sigma} \equiv 0,\left.\quad \frac{\partial w}{\partial v}\right|_{\Sigma} \equiv u ;\left.\quad \theta\right|_{\Sigma} \equiv 0,
\end{gathered}
$$

with $u \in L_{2}\left(0, T ; L_{2}(\Gamma)\right)$, and the regularity already obtained in (5.3) and (5.5) continuously in $u$. By superposition, we may consider two cases. In one case, we take $u \equiv 0$ in (5.52b) and replace $\theta_{t}$ with a function $f_{t}$ possessing the same regularity as $\theta_{t}$. The corresponding integrated-in-time version

$$
\begin{gathered}
\eta_{t t}-\gamma \Delta \eta_{t t}+\Delta^{2} \eta-\Delta \eta_{t}=f \in L_{2}\left(0, T ; H^{-1}(\Omega)\right) ; \\
\eta(0, \cdot)=0, \quad \eta_{t}(0, \cdot)=0 ; \\
\left.\eta\right|_{\Sigma} \equiv 0,\left.\quad \frac{\partial \eta}{\partial v}\right|_{\Sigma} \equiv 0
\end{gathered}
$$

where $f$ plays the role of $\theta$ and hence is assumed to have the same regularity of $\theta$, see (5.5) for $p=2$.

For problem (5.53) we already know that the map

$$
\begin{aligned}
& \left.f \in L_{2}\left(0, T ; H^{-1}(\Omega)\right) \longrightarrow \Delta \eta\right|_{\Sigma} \in L_{2}(\Sigma) \\
& \left.\Delta \eta_{t}\right|_{\Sigma} \in H^{-1,0}(\Sigma) \equiv H^{-1}\left(0, T ; L_{2}(\Gamma)\right),
\end{aligned}
$$

by (5.11), (5.12), and (5.13) [7], [4, equations (3.97)-(3.99), page 129; also equations (C.47)-(C.49), page 206]: this result takes care of the contribution of the right-hand side $\theta_{t}$ in (5.52a) in obtaining, a fortiori, (5.51). 
The second problem, definitely more challenging, will be handled below in Theorem 5.6(ii), (5.60).

Step 5. Consider the elastic Kirchhoff equation

$$
\begin{array}{ll}
z_{t t}-\gamma \Delta z_{t t}+\Delta^{2} z-\Delta z_{t}=0 & \text { in }(0, T] \times \Omega \equiv Q ; \\
z(0, \cdot)=0, \quad z_{t}(0, \cdot)=0 & \text { in } \Omega ; \\
\left.z\right|_{\Sigma}=0,\left.\quad \frac{\partial z}{\partial v}\right|_{\Sigma}=g & \text { in }(0, T] \times \Gamma \equiv \Sigma
\end{array}
$$

(as in (4.1)). We can then state the following result.

THEOREM 5.6. With reference to problem (5.55), the maps

(i)

$$
\begin{aligned}
g \in H^{1,1}(\Sigma) & \longrightarrow z \in C\left([0, T] ; H^{2}(\Omega) \cap H_{0}^{1}(\Omega)\right) \\
& \longrightarrow z_{t} \in C\left([0, T] ; H_{0}^{1}(\Omega)\right) \\
& \longrightarrow z_{t t} \in C\left([0, T] ; \tilde{L}_{2}(\Omega)\right) \\
& \left.\longrightarrow \Delta z\right|_{\Sigma} \in L_{2}(\Sigma)
\end{aligned}
$$

are continuous.

(ii) As a consequence of (i), the map

$$
\left.g \in L_{2}(\Sigma) \longrightarrow \Delta z\right|_{\Sigma} \in H^{-1,-1}(\Sigma)
$$

is continuous.

Proof. The interior regularity (5.56), (5.57), and (5.58) can be reduced to Theorem 4.11.

Proof of the trace regularity (5.59). This is based on three main steps:

(i) Consider the mixed problem (5.55). Then the multiplier $h \cdot \nabla z$ applied to (5.55a), with $h \in\left[C^{2}(\bar{\Omega})\right]^{n}$ a vector field such that $\left.h\right|_{\Sigma}=v$ on $\Gamma$, yields the following estimates:

$$
\begin{aligned}
\left\|\left.\Delta z\right|_{\Sigma}\right\|_{L_{2}(\Sigma)}^{2} \leq & \left|\int_{\Sigma} \frac{\partial \Delta z}{\partial v} \frac{\partial z}{\partial v} d \Sigma\right|+\mathcal{O}\left(\int_{0}^{T}\left\|\left\{z, z_{t}\right\}\right\|_{H^{2}(\Omega) \times H^{1}(\Omega)}^{2} d t\right) \\
\leq & \epsilon\left\|\left.\frac{\partial \Delta z}{\partial v}\right|_{\Sigma}\right\|_{H^{-1,-1}(\Sigma)}^{2} \\
& +\frac{1}{\epsilon}\|g\|_{H^{1,1}(\Sigma)}^{2}+\mathcal{O}\left(\int_{0}^{T}\left\|\left\{z, z_{t}\right\}\right\|_{H^{2}(\Omega) \times H^{1}(\Omega)}^{2} d t\right),
\end{aligned}
$$

for any positive $\epsilon>0$ arbitrary. 
Estimate (5.57) stems from the usual identity, as for example, in [7, 8, 13], [17, identity (A.1), page 1016], which is obtained by applying (5.55a) by $h \cdot \nabla z$, $\left.h\right|_{\Gamma}=v$, by use-in addition-also of the BC $(5.55 \mathrm{c})$.

(ii) Consider the mixed problem (5.55). The multiplier $h \cdot \nabla \Delta z$, applied to (5.55a), with $h \in\left[C^{2}(\bar{\Omega})\right]^{n},\left.h\right|_{\Gamma}=v$ on $\Gamma$, yields the following estimate:

$$
\left\|\left.\frac{\partial \Delta z}{\partial v}\right|_{\Sigma}\right\|_{L_{2}(\Sigma)}^{2} \leq C_{T}\left\|\left.\Delta z\right|_{\Sigma}\right\|_{H^{1,1}(\Sigma)}^{2}+\mathcal{O}\left(\int_{0}^{T}\left\|\left\{z, z_{t}\right\}\right\|_{H^{3}(\Omega) \times H^{2}(\Omega)}^{2} d t\right) .
$$

Estimate (5.62) stems from the usual identity, as for example, in [14, identity (A.5), page 97], which is obtained by multiplying (5.55a) by $h \cdot \nabla \Delta z,\left.h\right|_{\Gamma}=v$, by use, in addition, also of the BC $(5.55 \mathrm{c})$.

(iii) With reference to the mixed problem (5.52), let

$$
y=\Lambda_{\Sigma}^{-1} z
$$

where $\Lambda_{\Sigma}^{-1}$ denotes an operator of order $(-1)$ in the time variable and in the tangential space variable. Then, estimate (5.62) for $z$ becomes the following estimate for $y$ (one unit lower):

$$
\left\|\left.\frac{\partial \Delta y}{\partial v}\right|_{\Sigma}\right\|_{H^{-1,-1}(\Sigma)}^{2} \leq C_{T}\left\|\left.\Delta y\right|_{\Sigma}\right\|_{L_{2}(\Sigma)}^{2}+\mathcal{O}\left(\int_{0}^{T}\left\|\left\{y, y_{t}\right\}\right\|_{H^{2}(\Omega) \times H^{1}(\Omega)}^{2} d t\right),
$$

where, modulo lower-order terms due to commutators, $y$ satisfies the same elastic Kirchhoff equation as (5.55) for $z$. See [15] for shifting the regularity in the wave equation case.

(iv) Because of the last statement, we can say that problem (5.55) also satisfies estimate (5.64). Thus, inequality (5.64) with $y$ now replaced by $z$, once used on the right-hand side of (5.61) yields

$$
\begin{aligned}
\left\|\left.\Delta z\right|_{\Sigma}\right\|_{L_{2}(\Sigma)}^{2} \leq & \epsilon C_{T}\left\|\left.\Delta z\right|_{\Sigma}\right\|_{L_{2}(\Sigma)}^{2}+\frac{1}{\epsilon}\|g\|_{H^{1,1}(\Sigma)}^{2} \\
& +\odot_{\epsilon}\left(\int_{0}^{T}\left\|\left\{z, z_{t}\right\}\right\|_{H^{2}(\Omega) \times H^{1}(\Omega)}^{2} d t\right),
\end{aligned}
$$

from which we obtain

$$
\begin{aligned}
\left(1-\epsilon C_{T}\right)\left\|\left.\Delta z\right|_{\Sigma}\right\|_{L_{2}(\Sigma)}^{2} \leq & \frac{1}{\epsilon}\|g\|_{H^{1,1}(\Sigma)}^{2} \\
& +\sigma_{\epsilon}\left(\int_{0}^{T}\left\|\left\{z, z_{t}\right\}\right\|_{H^{2}(\Omega) \times H^{1}(\Omega)}^{2} d t\right)
\end{aligned}
$$

(by (5.56) and (5.57)) $\leq C_{\epsilon}\|g\|_{H^{1,1}(\Sigma)}^{2}$, 
where in the last step we have used (5.56) and (5.57). Thus, taking $\epsilon>0$ small, we conclude that (5.67) proves (5.58).

The proof of part (ii) of Theorem 5.6 can be given using shifting techniques as in [15].

\section{Acknowledgement}

This research is partially supported by the National Science Foundation under Grant DMS-9804056.

\section{References}

[1] J.-P. Aubin, Approximation of Elliptic Boundary-Value Problems, Pure and Applied Mathematics, vol. XXVI, Wiley-Interscience, New York, 1972. MR 57\#18139. $\mathrm{Zbl} 248.65063$.

[2] L. DeSimone, Un 'applicazione della teoria degli integrali singolari allo studio delle equazion differenzioli lineari astrette del primo ordine, Rendic. Semin. Matemat., Universitā di Padova 34 (1964), 205-223 (Italian).

[3] M. Eller, I. Lasiecka, and R. Triggiani, Simultaneous exact/approximate boundary controllability of thermo-elastic plates with variable thermal coefficient and moment control, J. Math. Anal. Appl. 251 (2000), no. 2, 452-478. CMP 1794432. Zbl 0968.35017.

[4] Simultaneous exact/approximate boundary controllability of thermo-elastic plates with variable transmission coefficient, Shape Optimization and Optimal Design (Cambridge, 1999), Lecture Notes in Pure and Applied Mathematics, vol. 216, Dekker, New York, 2001, pp. 109-230. CMP 1812362.

[5] J. R. Giles, Introduction to the Analysis of Normed Linear Spaces, Australian Math. Soc. Lecture Series, vol. 13, Cambridge University Press, Cambridge, 2000. MR 2000m:46001. Zbl 941.46001.

[6] P. Grisvard, Caractérisation de quelques espaces d'interpolation, Arch. Rational Mech. Anal. 25 (1967), 40-63 (French). MR 35\#4718. Zbl 187.05901.

[7] J. Lagnese and J.-L. Lions, Modelling Analysis and Control of Thin Plates, Research in Applied Mathematics, vol. 6, Masson, Paris, 1988. MR 89k:73001. Zbl 662.73039 .

[8] J. E. Lagnese, Boundary Stabilization of Thin Plates, SIAM Studies in Applied Mathematics, vol. 10, SIAM, Pennsylvania, 1989. MR 91k:73001. Zbl 696.73034.

[9] I. Lasiecka, Controllability of a viscoelastic Kirchhoff plate, Control and Estimation of Distributed Parameter Systems (Vorau, 1988) (Basel), Int. Series Num. Math., vol. 91, Birkhäuser, 1989, pp. 237-247. MR 90k:93012. Zbl 685.93007.

[10] I. Lasiecka and R. Triggiani, Sharp trace regularity of elastic and thermoelastic Kirchhoff equations with clamped B.C., preprint.

[11] Exact controllability of the wave equation with Neumann boundary control, Appl. Math. Optim. 19 (1989), no. 3, 243-290. MR 90a:49039. Zbl 666.49012.

[12] _ Differential and Algebraic Riccati Equations with Application to Boundary/Point Control Problems: Continuous Theory and Approximation Theory, 
Lecture Notes in Control and Information Sciences, vol. 164, Springer-Verlag, Berlin, 1991. MR 92k:93009. Zbl 754.93038.

[13] __ Exact controllability and uniform stabilization of Euler-Bernoulli equations with boundary control only in $\left.\Delta w\right|_{\Sigma}$, Boll. Un. Mat. Ital. B (7) 5 (1991), no. 3, 665-702. MR 92i:93013. Zbl 751.93006.

[14] Exact controllability and uniform stabilization of Kirchoff [Kirchhoff] plates with boundary control only on $\left.\Delta w\right|_{\Sigma}$ and homogeneous boundary displacement, J. Differential Equations 93 (1991), no. 1, 62-101. MR 92i:93014. Zbl 748.93010.

[15] Uniform stabilization of the wave equation with Dirichlet or Neumann feedback control without geometrical conditions, Appl. Math. Optim. 25 (1992), no. 2, 189-224. MR 93b:93099. Zbl 780.93082.

[16] _ Control Theory for Partial Differential Equations: Continuous and Approximation Theories. II, Abstract Hyperbolic-Like Systems over a Finite Time Horizon. Encyclopedia of Mathematics and Its Applications, vol. 75, Cambridge University Press, Cambridge, 2000. CMP 1745 476. Zbl 961.93003.

[17] Sharp regularity theory for elastic and thermoelastic Kirchoff [Kirchhoff] equations with free boundary conditions, Rocky Mountain J. Math. 30 (2000), no. 3, 981-1024. MR 2001i:35039.

[18] _ Structural decomposition of thermo-elastic semigroups with rotational forces, Semigroup Forum 60 (2000), no. 1, 16-66. CMP 1829930.

[19] J.-L. Lions and E. Magenes, Non-Homogeneous Boundary Value Problems and Applications. Vol. I, Springer-Verlag, New York, 1972. MR 50\#2670. Zbl 223.35039.

[20] A. E. Taylor and D. C. Lay, Introduction to Functional Analysis, 2nd ed., John Wiley \& Sons, New York, 1980. MR 81b:46001. Zbl 501.46003.

[21] R. Triggiani, Regularity with interior point control. II. Kirchhoff equations, J. Differential Equations 103 (1993), no. 2, 394-420. MR 94g:93068. Zbl 800.93596.

[22] Sharp regularity theory for thermo-elastic mixed problems, Appl. Anal. 73 (1999), no. 3-4, 557-572. MR 2000j:35258.

Irena Lasiecka: Department of Mathematics, University of Virginia, Charlottesville, VA 22904, USA

E-mail address: i12v@virginia.edu

Roberto Triggiani: Department of Mathematics, University of Virginia, Charlottesville, VA 22904, USA

E-mail address: rt7u@virginia.edu 


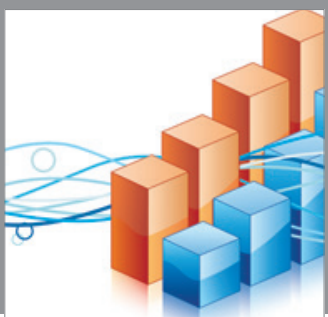

Advances in

Operations Research

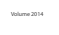

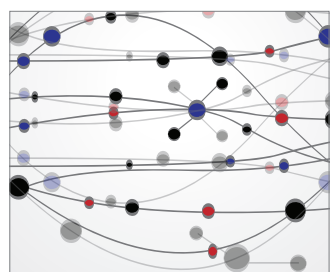

\section{The Scientific} World Journal
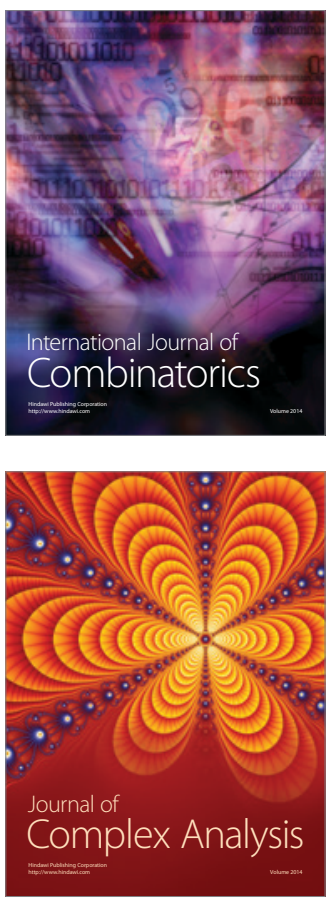

International Journal of

Mathematics and

Mathematical

Sciences
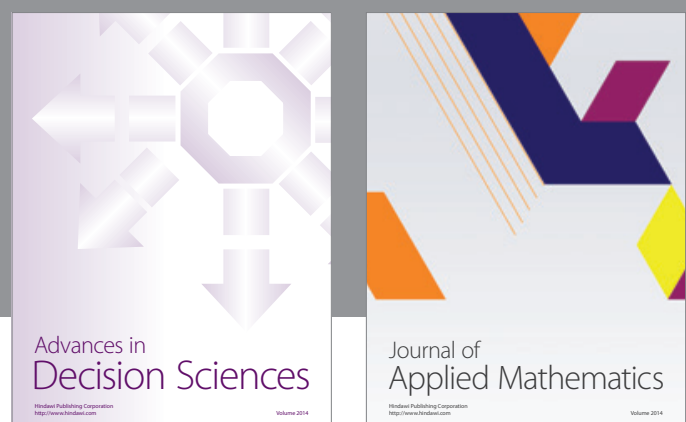

Journal of

Applied Mathematics
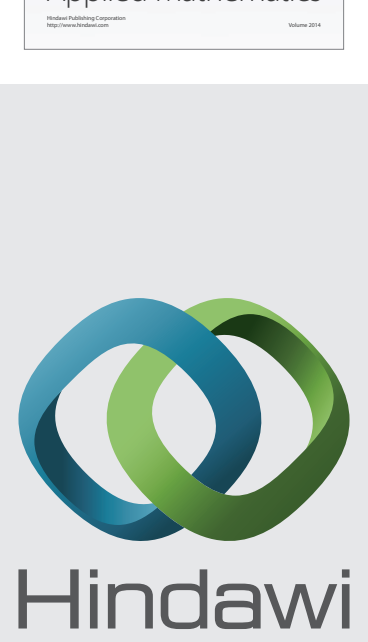

Submit your manuscripts at http://www.hindawi.com
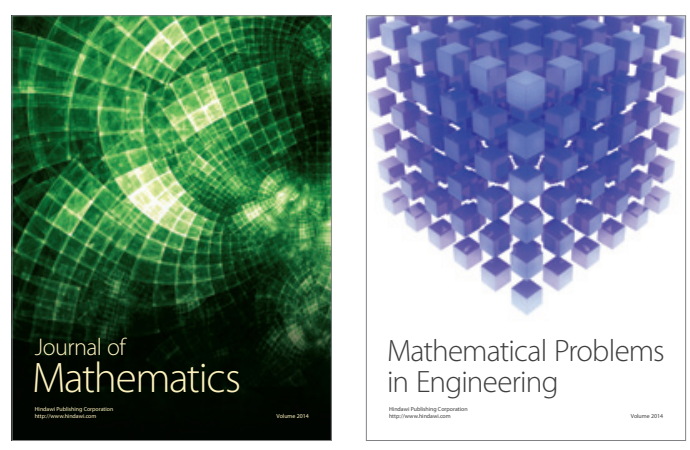

Mathematical Problems in Engineering
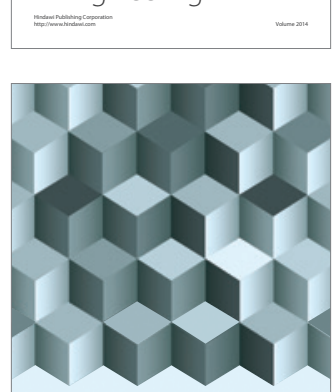

Journal of

Function Spaces
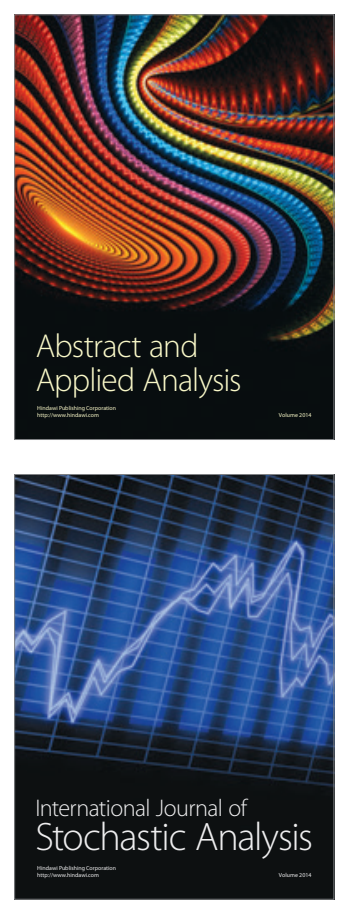

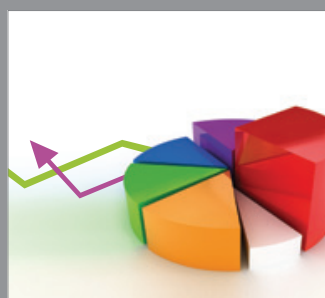

ournal of

Probability and Statistics

Promensencen
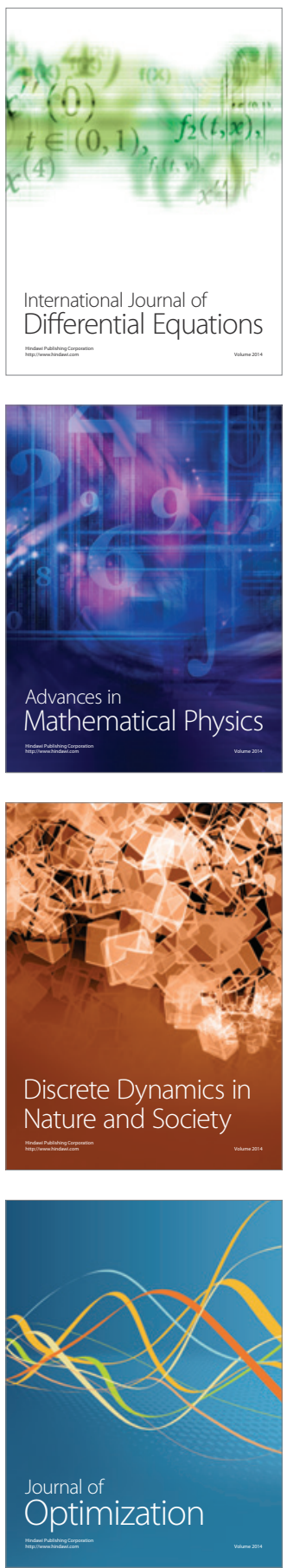